\title{
Higgs-dilaton cosmology: From the early to the late Universe
}

\author{
Juan García-Bellido, ${ }^{1, *}$ Javier Rubio, ${ }^{1, \dagger}$ Mikhail Shaposhnikov, ${ }^{2, \ddagger}$ and Daniel Zenhäusern ${ }^{2, \S}$ \\ ${ }^{1}$ Instituto de Física Teórica CSIC-UAM, Universidad Autónoma de Madrid, Cantoblanco 28049 Madrid, Spain \\ ${ }^{2}$ Institut de Théorie des Phénomènes Physiques, École Polytechnique Fédérale de Lausanne, CH-1015 Lausanne, Switzerland
}

(Received 5 August 2011; published 7 December 2011)

\begin{abstract}
We consider a minimal scale-invariant extension of the standard model of particle physics combined with unimodular gravity formulated in [M. Shaposhnikov and D. Zenhausern, Phys. Lett. B 671, 187 (2009).]. This theory is able to describe not only an inflationary stage, related to the standard model Higgs field, but also a late period of dark-energy domination, associated with an almost massless dilaton. A number of parameters can be fixed by inflationary physics, allowing us to make specific predictions for any subsequent period. In particular, we derive a relation between the tilt of the primordial spectrum of scalar fluctuations, $n_{s}$, and the present value of the equation of state parameter of dark energy (DE), $w_{\mathrm{DE}}^{0}$. We find bounds for the scalar tilt, $n_{s}<0.97$, the associated running, $-0.0006<d \ln n_{s} / d \ln k \lesssim$ -0.00015 , and for the scalar-to-tensor ratio, $0.0009 \lessgtr r<0.0033$, which will be critically tested by the results of the Planck mission. For the equation of state of dark energy, the model predicts $w_{\mathrm{DE}}^{0}>-1$. The relation between $n_{s}$ and $w_{\mathrm{DE}}^{0}$ allows us to use the current observational bounds on $n_{s}$ to further constrain the dark-energy equation of state to $0<1+w_{\mathrm{DE}}^{0}<0.02$, which is to be confronted with future dark-energy surveys.
\end{abstract}

DOI: 10.1103/PhysRevD.84.123504

PACS numbers: $98.80 . \mathrm{Cq}, 95.36 .+\mathrm{x}$

\section{INTRODUCTION}

At the classical level, the Lagrangian describing the standard model (SM) of particle physics minimally coupled to general relativity (GR) contains three dimensional parameters: Newton's constant $G$, the vacuum expectation value of the Higgs field or, equivalently, the Higgs boson mass and a possible cosmological constant $\Lambda$. The masses of quarks, leptons and intermediate vector bosons are induced by the vacuum expectation value of the Higgs field. At the quantum level, additional scales, such as $\Lambda_{\mathrm{QCD}}$ and all other scales related to the running of coupling constants, appear due to dimensional transmutation. It is tempting to look for models in which all these seemingly unrelated scales have a common origin.

In this work, we present a detailed analysis of a model realizing this idea, proposed in [1]. We will refer to it as the "Higgs-dilaton model." The model is based on a minimal extension of the SM and GR that contains no dimensional parameters in the action and is, therefore, scale-invariant at the classical level. Scale invariance is achieved by introducing a new scalar degree of freedom, called dilaton. The motivation of the model relies on the assumption that the structure of the theory is not changed at the quantum level. In other words, the full quantum effective action should still be scale-invariant, and the effective scalar potential should preserve the features of the classical potential. A perturbative quantization procedure maintaining scale invariance was presented in [2] (see also [3]). In the

\footnotetext{
*juan.garciabellido@uam.es

†javier.rubio@uam.es

mikhail.shaposhnikov@epfl.ch

$\S$ daniel.zenhaeusern@epfl.ch
}

Higgs-dilaton model, all scales are induced by the spontaneous breakdown of scale invariance (SI). As a consequence of the broken symmetry, the physical dilaton is exactly massless. Replacing GR by unimodular gravity (UG), in which the metric determinant is fixed to one, $|g|=1$, results in the appearance of an arbitrary integration constant in the equations of motion, representing an additional breaking of scale symmetry. As discussed in [1], in theories with scalar fields nonminimally coupled to gravity, this constant effectively gives rise to a nontrivial potential for the scalar fields. In the case of the Higgsdilaton model, the new potential is of the "run-away" type in the direction of the dilaton.

While the dynamical breakdown of the scale symmetry by the Higgs field can provide a mechanism for inflation in the early Universe [4], the light dilaton, practically decoupled from all SM fields, can act as quintessence $(\mathrm{QE})$, i.e. as dynamical dark energy (DE). We find that, under some assumptions, it is possible to relate the observables associated to inflation to those associated to dark energy. Namely, we establish a functional relation between the predicted value for the tilt $n_{s}$ of the primordial scalar power spectrum and the predicted equation of state parameter $w_{\mathrm{DE}}^{0}$ of dark energy. Further, we find a relation involving the corresponding second order quantities, i.e. the running $\alpha_{\zeta}$ of the spectral tilt and the rate of change $w_{\mathrm{DE}}^{a}$ of the DE equation of state.

The paper is organized as follows: In Sec. II A, we introduce and discuss the minimal scale-invariant extension of the standard model and general relativity. In Sec. II B, the idea of unimodular gravity is described and applied to the scale-invariant model. We then discuss the cosmology of the resulting Higgs-dilaton model. 
The inflationary period is studied in detail in Sec. III. The implications of the model for the late dark-energydominated stage are studied in Sec. IV. Finally, conclusions are presented in Sec. V. For completeness, an analysis of slow-roll inflation in the Jordan frame and the differences with respect to the Einstein frame are presented in the Appendix.

\section{THE HIGGS-DILATON MODEL}

In this section, we review the Higgs-dilaton model of [1], which consists of two moderate extensions of the standard model and general relativity (SM plus GR). In Sec. II A, we show how the introduction of a dilaton allows us to extend SM plus GR to a phenomenologically viable scale-invariant theory. After discussing the main properties of the resulting theory (II A 1), we discuss two naturalness issues, the cosmological constant problem and the gauge hierarchy problem, in the context of this model (II A 2). Next, we give some arguments in favor of a particular parameter choice corresponding to the absence of a cosmological constant (II A 3). In Sec. II B, the construction of the model is completed by replacing GR with UG. The qualitative picture of cosmology in the Higgs-dilaton model, as found in [1], is recalled in Sec. II C.

\section{A. Minimal scale-invariant extension of SM plus GR}

\section{Introducing the Dilaton}

Let us start by writing down the Lagrangian density that combines GR and the $\mathrm{SM}{ }^{1}$

$$
\frac{\mathcal{L}}{\sqrt{-g}}=\frac{1}{2} M_{P}^{2} R+\mathcal{L}_{\mathrm{SM}[\lambda \rightarrow 0]}-\lambda\left(\varphi^{\dagger} \varphi-v^{2}\right)^{2}-\Lambda,
$$

where the first term is the usual Einstein-Hilbert action for GR with $M_{P}=(8 \pi G)^{-1 / 2}$, the second term is the SM Lagrangian without the Higgs potential, the third term is the Higgs potential with the SM Higgs doublet $\varphi$ and its vacuum expectation value $v$, and $\Lambda$ is a cosmological constant. In this standard theory, to which we will refer as "SM plus GR", classical scale invariance is violated by the presence of the dimensional constants $M_{P}, v$ and $\Lambda$. Our goal is to let these scales be dynamical, i.e. replace them by a field. The most obvious solution, without introducing new degrees of freedom, would be to let the Higgs field be responsible for all scales. This corresponds to considering the Lagrangian ${ }^{2}$

$$
\frac{\mathcal{L}}{\sqrt{-g}}=\xi \varphi^{\dagger} \varphi R+\mathcal{L}_{\mathrm{SM}[\lambda \rightarrow 0]}-\lambda\left(\varphi^{\dagger} \varphi\right)^{2}
$$

\footnotetext{
${ }^{1}$ We use the conventions $\eta_{\mu \nu}=\operatorname{diag}(-1,1,1,1)$ and $R_{\beta \gamma \delta}^{\alpha}=$ $\partial_{\gamma} \Gamma_{\beta \delta}^{\alpha}+\Gamma_{\lambda \gamma}^{\alpha} \Gamma_{\beta \delta}^{\lambda}-(\gamma \leftrightarrow \delta)$.

${ }^{2}$ With the conventions used here, a conformally coupled scalar field has $\xi=-1 / 6$.
}

$\xi$ being a new real parameter ("nonminimal coupling"). The associated action is now scale-invariant, i.e. invariant under the global transformations,

$$
g_{\mu \nu}(x) \mapsto g_{\mu \nu}(\sigma x), \quad \Phi(x) \mapsto \sigma^{d_{\Phi}} \Phi(\sigma x),
$$

where $\Phi(x)$ stands for the different particle physics fields, $d_{\Phi}$ is their associated scaling dimension, and $\sigma$ is an arbitrary real parameter. In a theory that is invariant under all diffeomorphisms, as is the case for Eq. (2), the symmetry associated with the absence of dimensional parameters can equivalently be written as an internal transformation $^{3}$

$$
g_{\mu \nu}(x) \mapsto \sigma^{-2} g_{\mu \nu}(x), \quad \Phi(x) \mapsto \sigma^{d_{\Phi}} \Phi(x) .
$$

Can the Lagrangian (2) give a satisfactory phenomenology? Since we are looking for a theory that should eventually be quantized, we want to introduce the requirement that the theory possesses a classical ground state. The term "classical ground state" will be used throughout this work to refer to solutions of the classical equations of motion, which correspond to constant fields in the particle physics sector of the theory and a maximally symmetric geometry, i.e. Minkowski (flat), de Sitter (dS) or anti-de Sitter (AdS) space-time. The existence of such a ground state might be essential for a consistent quantization of the theory. At the quantum level, the theory should possess a ground state that breaks scale invariance, and in this way, induces masses and dimensional couplings for the excitations (particles). We will require that this spontaneous symmetry breaking already appears in the classical theory due to the existence of a symmetry-breaking classical ground state. ${ }^{4}$

Let us now look for symmetry-breaking classical ground states in the theory (2). If gravity is neglected, i.e. the first term in the Lagrangian is dropped, the classical ground states correspond to the minima of the scalar potential $\lambda\left(\varphi^{\dagger} \varphi\right)^{2}$. The only possibility for them to break the scale symmetry, $\varphi=\varphi_{0} \neq 0$, is to set $\lambda=0$. In this case, the theory possesses an infinite family of classical ground states satisfying $2 \varphi^{\dagger} \varphi=h_{0}^{2}$, where $h_{0}$ is an arbitrary real constant. If one includes gravity, the set of possible classical ground states becomes richer. Namely, even if $\lambda \neq 0$, the theory possesses a continuous family of classical ground states satisfying $2 \varphi^{\dagger} \varphi=h_{0}^{2}$ and $R=4 \lambda h_{0}^{2} / \xi$, where $h_{0}$ is an arbitrary real constant. The states with $h_{0} \neq 0$ break scale invariance spontaneously and induce all scales at the classical level. Hence, the goal of having a classical theory in which all scales have the same origin,

\footnotetext{
${ }^{3}$ Note that, in a theory that is invariant only under a restricted class of diffeomorphisms, such as UG, the absence of dimensional parameters will still guarantee invariance under Eq. (3) but not under Eq. (4).

${ }^{4}$ The authors of $[5,6]$ propose that scale symmetry could be broken by the pure presence of a time-dependent cosmological background.
} 
spontaneous breakdown of SI, is achieved. However, the above theory is in conflict with experimental constraints. In fact, although the nonzero background value of $\varphi$ gives masses to all other SM particles, the excitations of the Higgs field itself are massless and, moreover, decoupled from the SM fields [7]. This fact is seen most easily if the Lagrangian is written in the Einstein frame by defining the new metric $\tilde{g}_{\mu \nu}=M_{P}^{-2} \xi \varphi^{\dagger} \varphi g_{\mu \nu}$ and the new canonical Higgs field $\tilde{\varphi}=M_{P} \sqrt{1 / \xi+6} \ln \left(\varphi / M_{P}\right)$. (This type of variable change will be discussed in detail in Sec. III). In the new variables, the SI of the original formulation corresponds to a shift symmetry for the Higgs field $\tilde{\varphi}$, which is the massless Goldstone boson associated with the spontaneous breakdown of SI. A Higgs field with these properties is excluded by electroweak precision tests [8]. Therefore, in order to construct a viable SI theory, it seems unavoidable to introduce new degrees of freedom.

The next simplest possibility is to add a new singlet scalar field to the theory. We will refer to it as the dilaton $\chi$. The scale-invariant extension for the SM plus GR including the dilaton reads

$$
\begin{aligned}
\frac{\mathcal{L}_{\mathrm{SI}}}{\sqrt{-g}}= & \frac{1}{2}\left(\xi_{\chi} \chi^{2}+2 \xi_{h} \varphi^{\dagger} \varphi\right) R+\mathcal{L}_{\mathrm{SM}[\lambda \rightarrow 0]} \\
& -\frac{1}{2} g^{\mu \nu} \partial_{\mu} \chi \partial_{\nu} \chi-V(\varphi, \chi),
\end{aligned}
$$

where the scalar potential is given by ${ }^{5}$

$$
V(\varphi, \chi)=\lambda\left(\varphi^{\dagger} \varphi-\frac{\alpha}{2 \lambda} \chi^{2}\right)^{2}+\beta \chi^{4} .
$$

We will only consider positive values for $\xi_{\chi}$ and $\xi_{h}$, such that the coefficient in front of the scalar curvature is positive, whatever values the scalar fields take. The positivity of the nonminimal coupling parameters is, at the same time, the condition for positive definiteness of the scalar-field kinetic terms. By construction, the action associated with Eq. (5) is invariant under Eq. (3), respectively (4). The theory should possess a symmetry-breaking classical ground state with $\varphi=\varphi_{0} \neq 0$ and $\chi=\chi_{0} \neq 0$. The case $\varphi_{0}=0$ would correspond to a theory with no electroweak symmetry breaking, while the case $\chi_{0}=0$ would result in a theory with a massless Higgs field. Both these cases are phenomenologically unacceptable.

Let us again start by neglecting the gravitational part of the action. In its absence, the ground states correspond to the minima of the potential (6). It is easy to see that the only possibility to get a ground state satisfying $\varphi_{0} \neq 0$ and $\chi_{0} \neq 0$ is to have a potential with a flat direction, i.e. $\alpha>0$ and $\beta=0$, as well as $\lambda>0$ for stability. The corresponding family of classical ground states is given

\footnotetext{
${ }^{5}$ The parametrization chosen for the scalar potential assumes $\lambda \neq 0$. This only excludes the phenomenologically unacceptable case where a quartic term $\left(\varphi^{\dagger} \varphi\right)^{2}$ is absent.
}

by $2 \varphi^{\dagger} \varphi=h_{0}^{2}$ and $\chi=\chi_{0}$ with $h_{0}^{2}=\frac{\alpha}{\lambda} \chi_{0}^{2}$, where $\chi_{0}$ is an arbitrary real constant.

Like before, the inclusion of gravity results in the appearance of additional classical ground states for $\beta \neq 0$, given by

$$
h_{0}^{2}=\frac{\alpha}{\lambda} \chi_{0}^{2}+\frac{\xi_{h}}{\lambda} R, \quad R=\frac{4 \beta \lambda \chi_{0}^{2}}{\lambda \xi_{\chi}+\alpha \xi_{h}} .
$$

The solutions with $\chi_{0} \neq 0$ spontaneously break SI. All scales are induced and proportional to $\chi_{0}$. For instance, one can directly identify the Planck scale as

$$
M_{P}^{2}=\xi_{\chi} \chi_{0}^{2}+\xi_{h} h_{0}^{2}=\left(\xi_{\chi}+\xi_{h} \frac{\alpha}{\lambda}+\frac{4 \beta \xi_{h}^{2}}{\lambda \xi_{\chi}+\alpha \xi_{h}}\right) \chi_{0}^{2} .
$$

Depending on the value of $\beta$, the background corresponds to flat space-time $(\beta=0)$, de Sitter, or anti-de Sitter spacetime of constant scalar curvature $R$, corresponding to a cosmological constant

$$
\Lambda=\frac{1}{4} M_{P}^{2} R=\frac{\beta M_{P}^{4}}{\left(\xi_{\chi}+\frac{\alpha}{\lambda} \xi_{h}\right)^{2}+4 \frac{\beta}{\lambda} \xi_{h}^{2}} .
$$

The spectrum of perturbations around a symmetry-breaking solution contains the usual massless spin- 2 perturbation in the gravitational sector. The scalar sector contains an excitation with mass

$$
m^{2}=2 \alpha M_{P}^{2} \frac{\left(1+6 \xi_{\chi}\right)+\frac{\alpha}{\lambda}\left(1+6 \xi_{h}\right)}{\xi_{\chi}\left(1+6 \xi_{\chi}\right)+\xi_{h} \frac{\alpha}{\lambda}\left(1+6 \xi_{h}\right)}+\mathcal{O}(\beta),
$$

which will play the role of the physical SM Higgs field, plus a massless Goldstone boson (both perturbations are combinations of the fields $\chi$ and $h$ ). We use $h$ to denote the field $\varphi$ in the unitary gauge. Like in the standard Higgs mechanism, the excitations of the standard model fields get masses proportional to $h_{0}$. If one extends the SM by introducing right-handed neutrinos $[9,10]$, these neutrinos get induced masses proportional to $\chi_{0}{ }^{6}{ }^{6}$

In the described model, physics is completely independent of the value of $\chi_{0}$, as long as $\chi_{0} \neq 0$. This is because only dimensionless ratios of the different scales can be measured. Therefore, parameters of the model have to be chosen such that these ratios correspond to the measured ones. For instance, one should reproduce the hierarchies between the cosmological scale and the electroweak scale, i.e. $\Lambda / M_{P}^{4} \sim \mathcal{O}\left(10^{-120}\right)$, as well as the ratio between the electroweak and the gravitational scale $m^{2} / M_{P}^{2} \sim$ $\mathcal{O}\left(10^{-30}\right)$. We choose the parameter $\beta$ to be responsible for the first ratio and $\alpha$ for the second ratio. Therefore, these parameters have to take values satisfying $\beta \lll \alpha \lll 1$ and $\beta, \alpha \lll \xi_{\chi}$, and $\xi_{h}$. One then gets approximately

\footnotetext{
${ }^{6}$ Gauge invariance does not allow for couplings of $\chi$ to SM fields.
} 
$\Lambda / M_{P}^{4} \simeq \frac{\beta}{\xi_{\chi}^{2}}$ and $m^{2} / M_{P}^{2} \simeq \frac{2 \alpha}{\xi_{\chi}}$. Note that the order of magnitude relation $\sqrt{\Lambda} / m^{2} \sim m^{2} / M_{P}^{2}$ (or, equivalently, $\sqrt{\beta} \sim \alpha^{2}$ ) is reminiscent of the big number coincidence pointed out by Dirac [11]. However, the present model does not address the question about the origin of the big differences between theses scales, i.e. the smallness of $\alpha$ and $\beta$, nor does it explain their approximate relation. The nonminimal couplings $\xi_{\chi}$ and $\xi_{h}$ will be constrained by cosmological considerations, and $\lambda \lesssim \mathcal{O}(1)$, as it corresponds to the self-coupling of the Higgs field. Therefore, one can fix the values of $\alpha$ and $\beta$ that give the correct ratios. In the same fashion, one has to choose values for the SM Yukawa couplings that produce the observed mass ratios.

As the theory contains a new massless degree of freedom, the dilaton, one has to make sure that it does not contradict any experimental bounds. A detailed analysis of the interactions between this massless field and the SM fields is contained in [12]. Let us cite the relevant findings of that work. It turns out that, as a consequence of SI, the massless scalar field completely decouples from all SM fields except for the Higgs field. Since the massless field is the Goldstone boson associated with the broken scale symmetry, there exists a set of field variables in terms of which it couples to the physical Higgs field only derivatively. In addition, for an appropriate choice of field variables, these interactions appear as nonrenormalizable operators, suppressed by the scale $M_{P} / \xi_{h}$. The analysis of Sec. III will show that $\xi_{h} \sim 10^{5}$. The suppression scale of nonrenormalizable operators is therefore considerably lower than the Planck scale, but still much larger than all known Particle physics scales.

Other deviations from the SM appear as a consequence of the nonminimal couplings to gravity. In fact, the physical Higgs field, i.e. the field that couples to the SM degrees of freedom, is not $h$, but a combination of $h$ and $\chi$. It was shown in [12] that the resulting deviations from the SM are suppressed by the ratio $\mathrm{m}^{2} / M_{P}^{2}$ between the physical Higgs mass and the Planck mass, respectively, by the small parameter $\alpha$. While the new massless field hardly affects SM phenomenology, we will see that it might play an important role in cosmology.

At the classical level, the above theory successfully implements the idea that all scales are consequences of the spontaneous breaking of SI. All conclusions remain true if SI and the features of the potential can be maintained at the quantum level (in this context, see $[2,13,14]$ ). In that case, the presented model is a viable effective fieldtheory extension of the SM and GR.

\section{Naturalness issues}

The presented theory contains two important finetunings related to the very big differences between the Planck scale $M_{P}$, the electroweak scale $m$, and the cosmological scale $\Lambda$. At the quantum level, this can lead to two much-discussed naturalness issues. One of them is part of the cosmological constant problem. In standard SM plus GR the effective cosmological constant is the sum of a bare constant and radiative corrections proportional to the particle physics mass scales of the theory, e.g. the electroweak scale. Matching the effective cosmological constant with its observed value, tiny compared to, for instance, the electroweak scale, requires a tremendous fine-tuning of the bare cosmological constant. In the case of the scale-invariant theory discussed here, the situation is somewhat different. Exact SI forbids a term $\sqrt{-g} \Lambda$ in the action. Also, if the quantization procedure respects SI, such a term is not generated radiatively. However, as we saw above, due to the nonminimal couplings of the scalar fields to gravity, the cosmological constant is, in fact, associated with the term $\beta \chi^{4}$. Now, this term is not forbidden by scale invariance. Therefore, even if scale invariance can be maintained at the quantum level, the quantum effective potential will contain a term $\beta_{\text {eff }} \chi^{4}$, where $\beta_{\text {eff }}$ is a combination of the bare value of $\beta$ and other nondimensional couplings of the theory. These other couplings are generally much bigger than the value of $\beta_{\text {eff }}$ that corresponds to the observed cosmological constant. So, again, a strong fine-tuning is needed in order to keep $\beta_{\text {eff }}$ sufficiently small. This tells us that the cosmological constant problem also exists in an exactly scale-invariant theory of the type proposed here.

The second naturalness issue is related to the mass of the Higgs boson and is commonly called "gauge hierarchy problem". The problem is twofold. The effective field theory combining the SM with GR contains two extremely different mass scales, namely, the electroweak scale $v=$ $246 \mathrm{GeV}$ ( $v$ being the vacuum expectation value of the Higgs field) and the Planck scale $M_{P}=(8 \pi G)^{-1 / 2}=$ $2.44 \times 10^{18} \mathrm{GeV}$. It is considered unnatural to have such a huge difference between two scales of the same theory. This is the first part of the gauge hierarchy problem. In the considered type of scale-invariant theories, the big difference between the electroweak and the Planck scale remains unexplained.

The other part of the gauge hierarchy problem is related to the stability of the Higgs mass against radiative corrections (for a recent discussion see, e.g. [15]). Much like the cosmological constant, the mass of the Higgs field gets radiative corrections proportional to the other particle physics mass scales of the theory. The logic is the same as in the case of the cosmological constant. If there exists a particle physics scale much bigger than the electroweak scale, the measured value of the electroweak scale can only be explained by an important fine-tuning of parameters. In other words, if there exists a new particle physics scale between the electroweak scale $m$ and the Planck scale $M_{P}$, the "smallness" of the Higgs mass constitutes a serious theoretical issue. This issue still appears in an exactly scale-invariant theory with spontaneous breaking of the scale symmetry. 
If the theory contains no intermediate particle physics scale between $m$ and $M_{P}$, the situation is different. In that case, whether or not the Higgs mass should be expected to contain big radiative corrections of the order $M_{P}$ depends on the ultraviolet (UV) completion of the theory. At the level of the low-energy effective field theory, the UV properties can be encoded in the choice of the renormalization scheme. A renormalization scheme based on the assumption that the UV completion is scale-invariant, and which does not bring in extra particle physics scales, was presented in [2] (see also [3]). If this scheme is applied to the considered minimal scale-invariant extension of SM plus GR, the Higgs mass does not obtain corrections proportional to $M_{P}$ (induced by the vacuum expectation value of the dilaton), and there is no problem of stability of the Higgs mass against radiative corrections. Hence, SI makes for the absence of this part of the gauge hierarchy problem.

\section{The special case $\beta=0$}

We now want to give some arguments in favor of the case $\beta=0$. This case corresponds to the existence of a flat direction in the Jordan-frame potential (6) and, hence, to the absence of a cosmological constant.

The reasoning of the precedent paragraph tells us that choosing $\beta=0$ corresponds to a fine-tuning of the parameters, especially at the quantum level, just like putting $\Lambda=0$ in standard SM plus GR. From this point of view, such a parameter choice should clearly be disfavored. Nevertheless, we think that the case $\beta=0$ is specially interesting. One reason is that only if $\beta=0$, SI can be spontaneously broken in the absence of gravity. Put in other words, $\beta=0$ allows flat space-time together with $(\varphi, \chi)=\left(\varphi_{0}, \chi_{0}\right) \neq(0,0)$ to be a classical solution.

Another argument is related to the stability of the ground state. As discussed above, a scale-invariant theory with spontaneous symmetry breaking always contains a massless scalar degree of freedom, Goldstone boson, independently of the value of $\beta$. Now, if $\beta \neq 0$, the background space-time of the theory corresponds to de Sitter (or anti-de Sitter) space-time. It is known, however, that a massless scalar field is unstable in de Sitter space-time [16]. There are also indications that this is the case for the 4-dimensional AdS [17]. Therefore, it is conceivable that a consistent quantization of the theory might rely on the requirement $\beta=0$ and, hence, the existence of flat space-time as a solution (see also [18-22]).

A third aspect appears in the context of cosmology. The theory with $\beta=0$, not containing a cosmological constant, does not seem to withstand the confrontation with cosmological observations. Just like the case $\beta<0$ (AdS), it can not explain the observed accelerated expansion of the Universe without introduction of a new dark-energy component. From this point of view, the only viable option seems to be $\beta>0(\mathrm{dS})$. This conclusion is correct if gravity is described by GR. However, as we will see in the upcoming section, the situation is very different if GR in Eq. (5) is replaced by unimodular gravity. In that case, the appearance of an arbitrary integration constant will give rise to a potential for the Goldstone boson of broken scale invariance. As a consequence, for appropriate parameter values and initial conditions, the now pseudoGoldstone boson can act as a dynamical dark-energy component. In this new situation, the case $\beta=0$ will again be peculiar, because it is the only case where dark energy is purely dynamical and has no constant contribution.

Based on these reasons, in what follows, we will single out the case $\beta=0$ and study the associated phenomenology in more detail.

\section{B. Combining scale invariance and unimodular gravity}

We now want to add to the idea of SI the idea of UG [23-30] and apply it to the Higgs-dilaton scenario. In $\mathrm{UG}$, one reduces the independent components of the metric $g_{\mu \nu}$ by one, imposing that the metric determinant $g \equiv$ $\operatorname{det}\left(g_{\mu \nu}\right)$ takes some fixed constant value. Conventionally, one takes $|g|=1$, hence the name. Fixing the metric determinant to one is not a strong restriction, in the sense that the family of metrics satisfying this requirement can still describe all possible geometries.

If we impose the unimodular constraint, the scaleinvariant Lagrangian (5) becomes

$$
\begin{aligned}
\mathcal{L}_{\mathrm{SI}-\mathrm{UG}}= & \frac{1}{2}\left(\xi_{\chi} \chi^{2}+2 \xi_{h} \varphi^{\dagger} \varphi\right) \hat{R}+\hat{\mathcal{L}}_{\mathrm{SM}[\lambda \rightarrow 0]} \\
& -\frac{1}{2} \hat{g}^{\mu \nu} \partial_{\mu} \chi \partial_{\nu} \chi-V(\varphi, \chi),
\end{aligned}
$$

where a hat on a quantity, like $\hat{R}$, indicates that it depends on the unimodular metric $\hat{g}_{\mu \nu}$, which satisfies $\operatorname{det} \hat{g}_{\mu \nu}=-1$. The potential $V(\varphi, \chi)$ is still given by (6). As a consequence of the unimodular constraint, the action associated to $\mathcal{L}_{\mathrm{SI}-\mathrm{UG}}$ is no longer invariant under all diffeomorphisms, but only under transverse diffeomorphisms, i.e. coordinate transformations $x^{\mu} \mapsto x^{\mu}+\xi^{\mu}(x)$, with the condition $\partial_{\mu} \xi^{\mu}=0$. Just as in pure UG (not including nongravitational fields), the equations of motion derived from the Lagrangian (11) contain an arbitrary integration constant $\Lambda_{0}$, which can be interpreted as an additional initial condition. It was shown in [1] that the classical solutions obtained from the Lagrangian (11) are equivalent to the solutions derived from the equivalent diffeomorphism-invariant Lagrangian

$$
\begin{aligned}
\frac{\mathcal{L}_{e}^{\mathrm{SI}-\mathrm{UG}}}{\sqrt{-g}}= & \frac{1}{2}\left(\xi_{\chi} \chi^{2}+2 \xi_{h} \varphi^{\dagger} \varphi\right) R+\mathcal{L}_{\mathrm{SM}[\lambda \rightarrow 0]} \\
& -\frac{1}{2} g^{\mu \nu} \partial_{\mu} \chi \partial_{\nu} \chi-V(\varphi, \chi)-\Lambda_{0},
\end{aligned}
$$

where $\Lambda_{0}$ is the mentioned arbitrary constant. While in the original formulation (11), the dimensional constant $\Lambda_{0}$ only appears in the equations of motion and thereby 

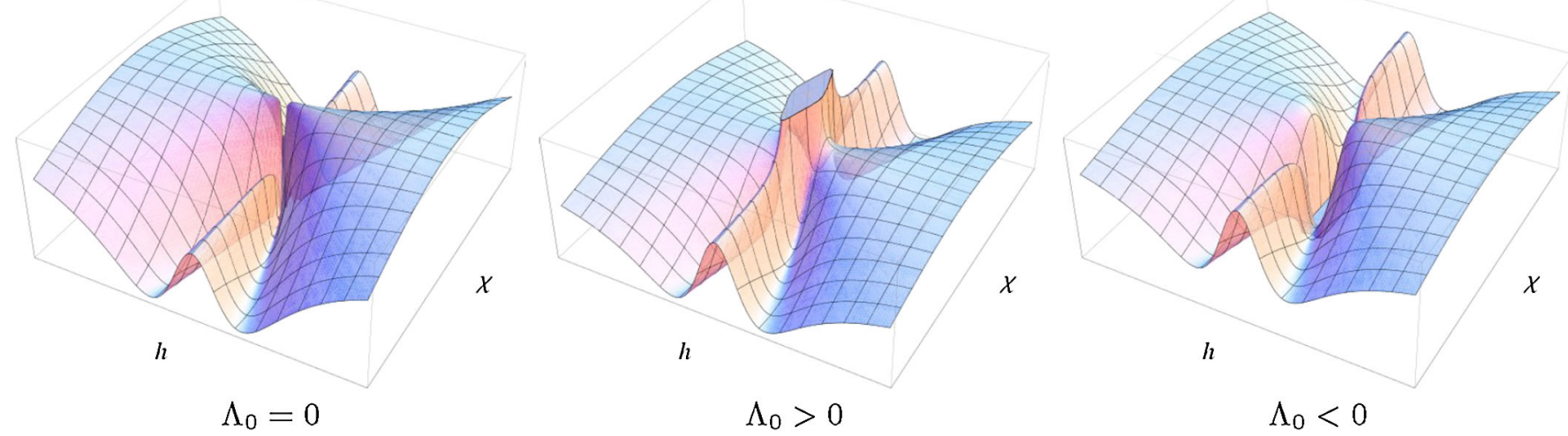

FIG. 1 (color online). These plots show the shape of the E-frame potential $\tilde{U}(h, \chi)$ (Eq. (18)) for $\Lambda_{0}=0, \Lambda_{0}>0$, and $\Lambda_{0}<0$, respectively.

spontaneously breaks SI; in the equivalent diffeomorphisminvariant formulation (12), the same constant appears as an explicit symmetry breaking in the action. Nevertheless, this constant should not be understood as a parameter in the action, but rather as an arbitrary initial condition. Given the equivalence of the two formulations, in order to study the phenomenology issued by Eq. (11), we will simply study the theory given by Eq. (12) for different values of $\Lambda_{0}{ }^{7}$

We now turn our attention to the physical implications of the term proportional to $\Lambda_{0}$. In the first instance, let us only consider the gravitational and the scalar sector of the theory, i.e.

$$
\begin{aligned}
\frac{\mathcal{L}}{\sqrt{-g}}= & \frac{1}{2}\left(\xi_{\chi} \chi^{2}+\xi_{h} h^{2}\right) R-\frac{1}{2}\left(\partial_{\mu} \chi\right)^{2} \\
& -\frac{1}{2}\left(\partial_{\mu} h\right)^{2}-V(h, \chi)-\Lambda_{0},
\end{aligned}
$$

where $h$ is the Higgs field in the unitary gauge. In order to simplify the physical interpretation, we define the Einstein frame (E-frame) metric ${ }^{8}$

$$
\tilde{g}_{\mu \nu}=M_{P}^{-2}\left(\xi_{\chi} \chi^{2}+\xi_{h} h^{2}\right) g_{\mu \nu}
$$

in terms of which the Lagrangian reads

$$
\frac{\mathcal{L}}{\sqrt{-\tilde{g}}}=M_{P}^{2} \frac{\tilde{R}}{2}-\frac{1}{2} \tilde{K}-\tilde{U}(h, \chi),
$$

where $\tilde{K}$ is a noncanonical but positive definite kinetic term (given below in Eq. (25)), and $\tilde{U}(h, \chi)$ is the E-frame potential given by

\footnotetext{
${ }^{7}$ Of course, the choice to analyze the theory in the diffeomorphism-invariant rather than in the original formulation is purely a matter of convenience.

${ }^{8}$ The Lagrangian in terms of the original variables is said to be written in the Jordan frame.
}

$$
\begin{aligned}
\tilde{U}(h, \chi)= & \frac{M_{P}^{4}}{\left(\xi_{\chi} \chi^{2}+\xi_{h} h^{2}\right)^{2}}\left(\frac{\lambda}{4}\left(h^{2}-\frac{\alpha}{\lambda} \chi^{2}\right)^{2}\right. \\
& \left.+\beta \chi^{4}+\Lambda_{0}\right) .
\end{aligned}
$$

Note that the E-frame potential gets singular at $\chi=h=0$. The reason is that, at this point, the conformal transformation (14) is singular, and the change to the E-frame is not allowed. Since for $\chi=h=0$ scale invariance is not broken, we will not be interested in the theory around this point. Let us discuss the shape of the E-frame potential and the classical ground states of the theory for $\alpha, \lambda, \xi_{\chi}$, and $\xi_{h}>0$ (cf. Figure 1). If $\Lambda_{0}=0$, in which case the theory (12) reduces to Eq. (5), the potential is minimal along the two valleys:

$$
h_{0}^{2}=\frac{\alpha}{\lambda} \chi_{0}^{2}+\frac{4 \beta \xi_{h} \chi_{0}^{2}}{\lambda \xi_{\chi}+\alpha \xi_{h}} .
$$

They correspond to the infinitely degenerate family of classical ground states found in Eq. (7). As before, if $\beta=0$, the potential vanishes at its minimum, while a nonzero $\beta$ gives rise to a cosmological constant (9). In other words, space-time in the classical ground state is Minkowskian, dS, or AdS. These are the results we have already discussed Sec. II A. As soon as $\Lambda_{0} \neq 0$, the valleys get a tilt, which lifts the degeneracy of the classical ground states. For $\Lambda_{0}<0$, the valleys are tilted towards the origin. The true classical ground state for this case is the trivial one, $\chi=h=0$. Hence, we discard this possibility. For $\Lambda_{0}>0$, the potential is tilted away from the origin; it is of the run-away type. In this case, the theory has an asymptotic classical ground state, given by Eq. (17) with $\chi_{0} \rightarrow \infty$. Again, depending on the value of $\beta$, this asymptotic solution corresponds to Minkowski, dS, or AdS space-time with curvature given by Eq. (9).

We see that, as a consequence of the nonminimal coupling between the scalar fields and gravity, the arbitrary integration constant $\Lambda_{0}$ does not play the role of a cosmological 
constant (as it does in pure UG), but rather gives rise to a peculiar potential for the scalar fields. For $\Lambda_{0}>0$, the potential is of the run-away type. In the following sections we will see that such a potential can have an interesting cosmological interpretation. In fact, the evolution of the scalar fields along the valley can give rise to dynamical dark energy (quintessence). We will focus on the case $\beta=0$, where dark energy does not contain a constant contribution and is purely due to the term proportional to $\Lambda_{0}$ (cf. arguments in Sec. II A 3).

While the term proportional to $\Lambda_{0}$ can play an important role in cosmology, its presence barely affects the particle physics phenomenology of the model. In fact, if the runaway potential is of the order of magnitude of the present dark-energy density, the time evolution of the background scalar fields along the valley can be neglected on particle physics time scales. Also, the additional interactions between the Higgs field and the dilaton that are induced by this potential are negligibly small.

\section{Higgs-dilaton cosmology - the qualitative picture}

In this subsection, we want to qualitatively describe the cosmological scenario issued by the model of Eq. (12) (or equivalently Eq. (11)) presented in the previous two subsections (cf. [1]). We consider the theory in the Einstein frame (15) and focus on the case $\beta=0$, for which the scalar-field potential (16) reduces to (cf. Figure 1)

$\tilde{U}(h, \chi)=\frac{M_{P}^{4}}{\left(\xi_{\chi} \chi^{2}+\xi_{h} h^{2}\right)^{2}}\left(\frac{\lambda}{4}\left(h^{2}-\frac{\alpha}{\lambda} \chi^{2}\right)^{2}+\Lambda_{0}\right)$.

The scalar fields $\chi$ and $h$ are now considered to be homogeneous background fields evolving in flat Friedmann-Lemaître-Robertson-Walker (FLRW) spacetime. Their evolution is affected by the noncanonical nature of the kinetic term. However, since the kinetic term is positive definite, in order to get a qualitative picture, it is enough to look at the features of the potential. In the absence of $\Lambda_{0}, \tilde{U}$ has its minima along the two valleys $h^{2}=\frac{\alpha}{\lambda} \chi^{2}$. The main effect of $\Lambda_{0} \neq 0$ is to give a tilt to the valleys. As discussed in the previous subsection, $\Lambda_{0}<0$ is phenomenologically unviable. We will only consider the case $\Lambda_{0}>0$, in which the valleys are tilted away from the origin.

For an appropriate choice of parameters, the crude picture of the role of the cosmological scalar fields is the following: If the scalar fields start off far from the valleys, $\Lambda_{0}$ can initially be neglected, and the scalar fields roll slowly towards one of the valleys. This roll-down can be responsible for cosmic inflation. As inflation is mainly driven by the Higgs field, this phase is much like in the case of the Higgs-Inflation model of [4].

After the end of inflation, preheating takes place. During this phase the scalar-field dynamics is dominated by the field $h$, and, hence, preheating in the present model is expected to be very similar as in the Higgs-Inflation model
[31,32] (see also [33]): The gauge bosons created at the minimum of the potential acquire a large mass while the Higgs field increases towards a maximal amplitude and starts to decay into all standard model leptons and quarks, rapidly depleting the occupation numbers of gauge bosons. The fraction of energy of the Higgs field that goes into SM particles is still very small compared to the energy contained in the oscillations, and, therefore, the nonperturbative decay is slow. As the Universe expands in a matterlike stage with zero pressure, the amplitude of the Higgs field oscillations decreases. Eventually, this amplitude is small enough so that the gauge boson masses become too small to induce a quick decay of the gauge bosons. As a consequence, their occupation numbers start to grow very rapidly via parametric amplification. After about a hundred oscillations, the produced gauge bosons backreact on the Higgs field, and the resonant production of particles stops. The Higgs field acquires a large mass via its interaction with the gauge condensate and preheating ends. From there on, the Higgs field, as well as the gauge fields, decay perturbatively until their energy is transferred to SM particles.

The phase of preheating is followed by the usual radiation- and matter-dominated stages, during which the scalar fields are "frozen" at some point of the valley. Their energy density is now given by the $\Lambda_{0}$ term and practically constant. As a consequence, it eventually comes to dominate over radiation and matter and, hence, provides a darkenergy component. In other words, the scalar fields rolling slowly down the potential valley play the role of a thawing quintessence field [34-37]. In this late stage, the fields satisfy $h(t)^{2} \simeq \frac{\alpha}{\lambda} \chi(t)^{2}$. On particle physics time scales the time variation of the background fields can be neglected. Perturbations around this almost-constant symmetrybreaking background can be interpreted as the SM particles plus an additional almost massless and almost decoupled particle, the dilaton. Note that as long as the background is constant, it is equivalent to quantize perturbations in the original (Jordan) frame or in the Einstein frame (cf. [38,39]).

In the following sections, we present a detailed analysis of the inflationary phase (Sec. IV) and the dark-energy dominated phase (Sec. IV). A detailed study of preheating in the present model is left for a future work.

Allowing for $\beta \neq 0$ in the potential would not affect the discussion of inflation. It will, however, have an effect on the dark-energy phenomenology, on which we will comment in Sec. IV.

\section{HIGGS-DILATON INFLATION}

As usual, it is assumed that during inflation all the energy of the system is contained in the inflaton fields and in the gravitational field. Therefore, during this stage, the SM fields can be neglected. Let us rewrite the scalartensor part of (12) as 


$$
\frac{\mathcal{L}}{\sqrt{-g}}=\frac{f(\phi)}{2} R-\frac{1}{2} g^{\mu \nu} \delta_{a b} \partial_{\mu} \phi^{a} \partial_{\nu} \phi^{b}-U(\phi),
$$

with a nonminimal coupling

$$
f(\phi) \equiv \sum_{a} \xi_{a} \phi^{a 2},
$$

and the potential

$$
U(\phi)=V(\phi)+\Lambda_{0}=\frac{\lambda}{4}\left(h^{2}-\frac{\alpha}{\lambda} \chi^{2}\right)^{2}+\Lambda_{0},
$$

including the SI breaking term $\Lambda_{0}$. As discussed in Sec. II A, the parameter $\alpha$ is set to be very tiny $\alpha \sim$ $\mathcal{O}\left(10^{-30}\right)$, in order to obtain the correct hierarchy between the electroweak and the Planck scale. Greek indices $\mu$, $\nu, \ldots=0,1,2,3$ denote space-time coordinates, while Latin indices are used to label the two real scalar fields present in the model: the dilaton field $\phi^{1}=\chi$ and the Higgs field in the unitary gauge $\phi^{2}=h$. The abstract notation in terms of $\phi^{i}$ will, in the following, allow us to interpret the scalar fields as the coordinates of a twodimensional sigma-model manifold. We will be able to write expressions and equations that are covariant under variable changes $\phi \mapsto \phi^{\prime}(\phi)$.

Whenever the nonminimal coupling is nonzero 9 $f(\phi) \neq 0$, one can define the new metric

$$
\tilde{g}_{\mu \nu}=\Omega^{2} g_{\mu \nu},
$$

with $\Omega^{2}=M_{P}^{-2} f(\phi)$ to reformulate the Lagrangian in the E-frame. Taking into account that the metric determinant and the Ricci scalar transform as ${ }^{10}$

$$
\begin{gathered}
\sqrt{-g}=\Omega^{-4} \sqrt{-\tilde{g}}, \\
R=\Omega^{2}\left(\tilde{R}+6 \tilde{\square} \ln \Omega-6 \tilde{g}^{\mu \nu} \partial_{\mu} \ln \Omega \partial_{\nu} \ln \Omega\right),
\end{gathered}
$$

one obtains

$$
\frac{\mathcal{L}}{\sqrt{-\tilde{g}}}=\frac{M_{P}^{2}}{2} \tilde{R}-\frac{1}{2} \tilde{K}-\tilde{U}(\phi),
$$

where the kinetic term is given by

$$
\tilde{K}=\gamma_{a b} \tilde{g}^{\mu \nu} \partial_{\mu} \phi^{a} \partial_{\nu} \phi^{b},
$$

and $\gamma_{a b}$ is a generally noncanonical and nondiagonal field-space metric, which, in terms of the variables $\left(\phi^{1}, \phi^{2}\right)=(\chi, h)$, is given by

$$
\gamma_{a b}=\frac{1}{\Omega^{2}}\left(\delta_{a b}+\frac{3}{2} M_{P}^{2} \frac{\Omega_{, a}^{2} \Omega_{, b}^{2}}{\Omega^{2}}\right) .
$$

\footnotetext{
${ }^{9}$ For our choice of parameters, where $\xi_{\chi}, \xi_{h}>0$ this is the case whenever the scalar fields are away from the origin $(\chi, h) \neq$ $(0,0)$.

${ }^{10}$ The action of the covariant d'Alembertian $\tilde{\square}$ on a scalar field $s(x)$ is given by $\tilde{\square} s=\frac{1}{\sqrt{-\tilde{g}}} \partial_{\mu}\left(\sqrt{-\tilde{g}} \tilde{g}^{\mu \nu} \partial_{\nu} s\right)$.
}

Unlike in the single-field case, the noncanonical kinetic term cannot, in general, be recast in canonical form by redefining the scalar-field variables. In fact, the field-space metric can be brought to canonical form by a local variable change if and only if its Riemann tensor identically vanishes. In the present case of a 2-dimensional manifold, the Riemann tensor has only one independent component, and it is enough to compute the Ricci scalar $R_{\gamma}$ associated with the field-space metric $\gamma_{a b}$,

$R_{\gamma}=\left(\xi_{h}-\xi_{\chi}\right) \frac{2}{M_{P}^{2}} \frac{\xi_{\chi}^{2}\left(1+6 \xi_{\chi}\right) \chi^{4}-\xi_{h}^{2}\left(1+6 \xi_{h}\right) h^{4}}{\left(\xi_{h}\left(1+6 \xi_{h}\right) h^{2}+\xi_{\chi}\left(1+6 \xi_{\chi}\right) \chi^{2}\right)^{2}}$.

For $R_{\gamma}$ to vanish globally, one would need to have $\xi_{\chi}=\xi_{h}$. As we will see, this case is not allowed by phenomenology. The E-frame potential is defined as

$$
\tilde{U}(\phi)=\tilde{V}(\phi)+\tilde{V}_{\Lambda_{0}}(\phi),
$$

where we have defined a scale-invariant and a scaleinvariance breaking part as

$$
\tilde{V}(\phi)=\frac{V(\phi)}{\Omega^{4}} \quad \text { and } \quad \tilde{V}_{\Lambda_{0}}(\phi)=\frac{\Lambda_{0}}{\Omega^{4}} .
$$

We can now write down the equations of motion derived from the E-frame Lagrangian (25). Einstein's equations are

$$
\begin{aligned}
\tilde{G}_{\mu \nu}= & \gamma_{a b}\left(\partial_{\mu} \phi^{a} \partial_{\nu} \phi^{b}-\frac{1}{2} \tilde{g}_{\mu \nu} \tilde{g}^{\rho \sigma} \partial_{\rho} \phi^{a} \partial_{\sigma} \phi^{b}\right) \\
& +\tilde{U} \tilde{g}_{\mu \nu},
\end{aligned}
$$

where $\tilde{G}_{\mu \nu}$ is the Einstein tensor computed from the metric $\tilde{g}_{\mu \nu}$. The equations for the scalar fields are

$$
\tilde{\square} \phi^{c}+\tilde{g}^{\mu \nu} \Gamma_{a b}^{c} \partial_{\mu} \phi^{a} \partial_{\nu} \phi^{b}=\tilde{U}^{; c},
$$

where $\Gamma_{a b}^{c}$ is the Christoffel symbol computed from the field-space metric $\gamma_{a b}$,

$$
\Gamma_{a b}^{c}=\frac{1}{2} \gamma^{c d}\left(\gamma_{d a, b}+\gamma_{d b, a}-\gamma_{a b, d}\right),
$$

and where we use the notation $\tilde{U}^{i c}=\gamma^{c d} \tilde{U}_{, d}$. Notice that Eqs. (31) and (32) are covariant under redefinitions of the scalar-field variables $\phi^{i} \mapsto \phi^{\prime i}(\phi)$.

We choose to do our analysis in the Einstein, rather than in the Jordan frame. The reason for this choice is that, in the literature predictions, for measurable quantities are usually computed in the Einstein frame, where gravity has the standard GR form. At the classical level, there is, apart from such practical arguments, nothing that would privilege one or the other frame. After all, the choice of the frame simply corresponds to a choice of variables.

\section{A. Exploiting scale invariance}

By construction, all terms in the Lagrangian (19), except the one proportional to $\Lambda_{0}$, are invariant under the scale transformations (4). We will see that if the $\Lambda_{0}$ term is to be 
associated with dark energy, it must be negligibly small during inflation. The approximate scale invariance of the theory will considerably simplify the analysis of the inflationary period.

\section{The Noether current of scale invariance}

Let us start by computing the Noether current associated with the scale transformations (4), which for an infinitesimal value of the parameter $\sigma$ become

$$
g_{\mu \nu} \mapsto g_{\mu \nu}+\sigma \Delta g_{\mu \nu}, \quad \phi^{i} \mapsto \phi^{i}+\sigma \Delta \phi^{i} .
$$

The explicit expressions for $\Delta g_{\mu \nu}$ and $\Delta \phi^{i}$ depend on the choice of the field variables. For the original variables, one has $\Delta g_{\mu \nu}=-2 g_{\mu \nu}, \Delta \chi=\chi$, and $\Delta h=h$. The associated current is given by (see, e.g. [40])

$$
\sqrt{-g} J^{\mu}=\frac{\partial \mathcal{L}}{\partial\left[\partial_{\mu} g_{\alpha \beta}\right]} \Delta g_{\alpha \beta}+\frac{\partial \mathcal{L}}{\partial\left[\partial_{\mu} \phi^{i}\right]} \Delta \phi^{i}
$$

and satisfies

$$
D_{\mu} J^{\mu}=-\frac{1}{\sqrt{-g}} \frac{\partial\left[\Lambda_{0} \sqrt{-g}\right]}{\partial g_{\mu \nu}} \Delta g_{\mu \nu}=4 \Lambda_{0},
$$

where $D_{\mu}$ denotes the covariant derivative constructed with the metric $g_{\mu \nu}$.

In the E-frame, scale transformations do not act on the metric, $\Delta \tilde{g}_{\mu \nu}=0$, and are simply given by

$$
\phi^{i} \mapsto \phi^{i}+\sigma \Delta \phi^{i} .
$$

In this case, the expression for the current is

$$
\sqrt{-\tilde{g}} \tilde{J}^{\mu}=\frac{\partial \mathcal{L}}{\partial\left[\partial_{\mu} \phi^{i}\right]} \Delta \phi^{i},
$$

while the conservation law becomes

$$
\tilde{D}_{\mu} \tilde{J}^{\mu}=-\frac{\partial \tilde{V}_{\Lambda_{0}}}{\partial \phi^{i}} \Delta \phi^{i}=\frac{4 \Lambda_{0}}{\Omega^{4}},
$$

where the covariant derivative $\tilde{D}_{\mu}$ is constructed with the metric $\tilde{g}_{\mu \nu}$. Whenever $\Lambda_{0}$ vanishes, scale invariance becomes exact, and the associated current $J^{\mu}$, or, equivalently, $\tilde{J}^{\mu}$, is conserved.

\section{New variables}

The approximate conservation law can lead us to a very convenient choice for the scalar-field variables in the E-frame formulation. In fact, one can always choose a set of variables $\left(\phi^{\prime 1}, \phi^{\prime 2}\right)=(\rho, \theta)$ such that a scale transformation only acts on a single variable $\rho$. Moreover, $\rho$ can always be defined such that the scale transformation acts on it like a shift $\rho \mapsto \rho+\sigma M_{P}$. This transformation is a symmetry of the Lagrangian if $\Lambda_{0}=0$. This means that $\rho$ can appear in the Lagrangian only through $\tilde{V}_{\Lambda_{0}}$ and through derivatives in the kinetic term. Moreover, the variable $\theta$ can be defined such that the field-space metric $\gamma_{a b}^{\prime}$, which is independent of $\rho$, is diagonal. If the Lagrangian (25) is expressed in terms of variables that satisfy these requirements, the current $\tilde{J}^{\mu}$ takes the form

$$
\tilde{J}^{\mu}=M_{P} \tilde{g}^{\mu \nu} \gamma_{\rho \rho}^{\prime}(\theta) \partial_{\nu} \rho .
$$

Now, in order to find the relation between the variables $\left(\phi^{\prime 1}, \phi^{\prime 2}\right)=(\rho, \theta)$ and the original variables $\left(\phi^{1}, \phi^{2}\right)=$ $(\chi, h)$, let us express the current $\tilde{J}^{\mu}$ in terms of the original variables

$\tilde{J}^{\mu}=\tilde{g}^{\mu \nu} \frac{M_{P}^{2}}{2\left(\xi_{\chi} \chi^{2}+\xi_{h} h^{2}\right)} \partial_{\nu}\left(\left(1+6 \xi_{\chi}\right) \chi^{2}+\left(1+6 \xi_{h}\right) h^{2}\right)$.

Comparing this expression to Eq. (40), it is clear that $\rho$ must be a function of the combination of the fields $\chi$ and $h$ acted upon by the partial derivative, i.e. $\rho=$ $\rho\left[\left(1+6 \xi_{\chi}\right) \chi^{2}+\left(1+6 \xi_{h}\right) h^{2}\right]$. For the scale transformation to correspond to a shift of $\rho$ by $\sigma M_{P}$, this function has to be chosen as

$$
\rho=\frac{M_{P}}{2} \ln \left(\frac{\left(1+6 \xi_{\chi}\right) \chi^{2}+\left(1+6 \xi_{h}\right) h^{2}}{M_{P}^{2}}\right) .
$$

The variable $\theta$, as it does not transform under scale transformations, has to be a function of the ratio between $h$ and $\chi$, i.e. $\theta=\theta\left[\frac{h}{\chi}\right]$. There is some freedom in the choice of this function. One can notice that the argument of the logarithm in Eq. (42) corresponds to the radius of an ellipse in the $(\chi, h)$-plane. We will therefore define $\theta$ as the angular coordinate of the ellipse, i.e.

$$
\theta=\arctan \left(\sqrt{\frac{1+6 \xi_{h}}{1+6 \xi_{\chi}}} \frac{h}{\chi}\right) .
$$

Let us note that, in terms of the variables $(\rho, \theta)$, since one has $\Delta \rho=M_{P}$ and $\Delta \theta=0$, the current conservation law (39) corresponds to the equation of motion for $\rho$. Further, one can see from Eq. (39) that the dependence of $\tilde{V}_{\Lambda_{0}}$ on $\rho$ is such that $\tilde{V}_{\Lambda_{0}} \propto \exp \left(-4 \rho / M_{P}\right)$.

In terms of the new variables, the E-frame kinetic term $\tilde{K}$ and the potential $\tilde{U}=\tilde{V}+\tilde{V}_{\Lambda_{0}}$ (cf. Eq. (25)) are given by

$$
\begin{aligned}
\tilde{K}= & \left(\frac{1+6 \xi_{h}}{\xi_{h}}\right) \frac{1}{\sin ^{2} \theta+\cos ^{2} \theta}(\partial \rho)^{2} \\
& +\frac{M_{P}^{2} \varsigma}{\xi_{\chi}} \frac{\tan ^{2} \theta+\mu}{\cos ^{2} \theta\left(\tan ^{2} \theta+\varsigma\right)^{2}}(\partial \theta)^{2}
\end{aligned}
$$

and

$$
\begin{gathered}
\tilde{V}(\theta)=\frac{\lambda M_{P}^{4}}{4 \xi_{h}^{2}}\left(\frac{\sin ^{2} \theta-\frac{\alpha}{\lambda} \frac{1+6 \xi_{h}}{1+6 \xi_{x}} \cos ^{2} \theta}{\sin ^{2} \theta+\operatorname{sos}^{2} \theta}\right)^{2}, \\
\tilde{V}_{\Lambda_{0}}(\rho, \theta)=\Lambda_{0}\left(\frac{1+6 \xi_{h}}{\xi_{h}}\right)^{2} \frac{e^{-4 \rho / M_{P}}}{\left(\sin ^{2} \theta+\operatorname{sos}^{2} \theta\right)^{2}},
\end{gathered}
$$


where we have defined the parameters

$$
\mu \equiv \frac{\xi_{\chi}}{\xi_{h}}, \quad \varsigma \equiv \frac{\left(1+6 \xi_{h}\right) \xi_{\chi}}{\left(1+6 \xi_{\chi}\right) \xi_{h}} .
$$

We will see in Sec. III D that for a successful description of inflation, the parameters have to be such that $\xi_{\chi} \sim$ $\mathcal{O}\left(10^{-3}\right)$ and $\xi_{h} \sim \mathcal{O}\left(10^{5}\right)$, and, hence, $\mu \ll 1$. In this case, one can neglect $\mu$ in the kinetic term (44). In this approximation, the action can be further simplified by introducing the variables

$$
\tilde{\rho}=\gamma^{-1} \rho \quad \text { and } \quad \tilde{\theta}=\frac{M_{P}}{a} \tanh ^{-1}(\sqrt{1-\varsigma} \cos \theta),
$$

with the parameters

$$
a=\sqrt{\frac{\xi_{\chi}(1-\varsigma)}{\varsigma}}, \quad \gamma=\sqrt{\frac{\xi_{\chi}}{1+6 \xi_{\chi}}} .
$$

In terms of these variables and for $\mu \ll 1$, the kinetic term takes the simple form

$$
\tilde{K} \simeq e^{2 b(\tilde{\theta})}(\partial \tilde{\rho})^{2}+(\partial \tilde{\theta})^{2},
$$

with

$$
b(\tilde{\theta})=\frac{1}{2} \ln \left(\operatorname{scosh}^{2}\left(a \tilde{\theta} / M_{P}\right)\right),
$$

which has been studied in the literature previously [41-43]. The potentials are given by

$$
\begin{gathered}
\tilde{V}(\tilde{\theta})=\frac{\lambda M_{P}^{4}}{4 \xi_{h}^{2}(1-\varsigma)^{2}}\left(1-\operatorname{scosh}^{2}\left(a \tilde{\theta} / M_{P}\right)\right. \\
\left.-\frac{\alpha}{\lambda} \frac{1+6 \xi_{h}}{1+6 \xi_{\chi}} \sinh ^{2}\left(a \tilde{\theta} / M_{P}\right)\right)^{2}, \\
\tilde{V}_{\Lambda_{0}}(\tilde{\rho}, \tilde{\theta})=\frac{\Lambda_{0}}{\gamma^{2}} \varsigma^{2} \cosh ^{4}\left(a \tilde{\theta} / M_{P}\right) e^{-4 \gamma \tilde{\rho} / M_{P}} .
\end{gathered}
$$

\section{Departure from scale invariance}

Let us now look at the E-frame equations of motion (31) and (32) in order to see in which region of field space the effect of a nonzero $\Lambda_{0}$ will be important. $\Lambda_{0}$ enters the equations through $\tilde{U}=\tilde{V}+\tilde{V}_{\Lambda_{0}}$ and through $\tilde{U}^{; c}=$ $\tilde{V}^{; c}+\tilde{V}_{\Lambda_{0}}^{; c}$. We therefore define the two new parameters

$$
\begin{gathered}
v_{1}=\frac{\tilde{V}_{\Lambda_{0}}}{\tilde{V}}, \\
v_{2}=\frac{\sqrt{\tilde{V}_{\Lambda_{0} ; a} \tilde{V}_{\Lambda_{0} ; a}}}{\sqrt{\tilde{V}^{; b} \tilde{V}_{; b}}}
\end{gathered}
$$

that characterize the departure from scale invariance. The parameter $v_{1}$ compares the importance of the scaleinvariance-breaking part of the potential $\tilde{V}_{\Lambda_{0}}$ to the scale-invariant part $\tilde{V}$ in the Einstein equations (31). In the region of field space where $v_{1} \ll 1, \tilde{V}_{\Lambda_{0}}$ can be neglected in Einstein's equations. The parameter $v_{2}$ provides a coordinate invariant measure of the importance of $\tilde{V}_{\Lambda_{0}} ; a$ compared to $\tilde{V}^{i a}$ in the scalar field equations (32). In fact, locally one can always choose a coordinate system in which $\left|\tilde{V}_{\Lambda_{0}} ; 1 / \tilde{V}^{; 1}\right|=\left|\tilde{V}_{\Lambda_{0}} ; 2 / \tilde{V}^{; 2}\right|=v_{2}$. Hence, in the region of field space where $v_{2} \ll 1, \tilde{V}_{\Lambda_{0}} ; a$ can be neglected in the scalar field equations. In the region where both $v_{1} \ll 1$ and $v_{2} \ll 1$ hold, the effect of $\Lambda_{0}$ is negligible, and the equations of motion become practically scale-invariant. We will refer to this region as the scale-invariant region. It will turn out that for phenomenologically viable values of the parameters, the whole period of observable inflation takes place in this region.

\section{B. Evolution of the homogeneous background}

Let us now consider homogeneous scalar fields $\phi^{i}=$ $\phi^{i}(t)$ in spatially flat FLRW space-time characterized by the line element

$$
d s^{2}=\tilde{g}_{\mu \nu} d x^{\mu} d x^{\nu}=-d t^{2}+a^{2}(t) d \vec{x}^{2} .
$$

Before writing down the equations of motion, we introduce some notation for vectors lying in the tangent and cotangent bundles of the field manifold that will allow us to write many of the upcoming expressions in a very compact way. The notation corresponds to the one of [44]. We denote vectors in boldface, i.e. $\boldsymbol{A}=\left(A^{1}, A^{2}\right)$. The inner product of two vectors $\boldsymbol{A}$ and $\boldsymbol{B}$ is given by

$$
\boldsymbol{A} \cdot \boldsymbol{B} \equiv \boldsymbol{A}^{\dagger} \boldsymbol{B}=\gamma_{i j} A^{i} B^{j},
$$

and the norm of a vector $\boldsymbol{A}$ is

$$
|A| \equiv \sqrt{A \cdot A},
$$

where a dagger $\dagger$ on a naturally contravariant or covariant vector denotes its dual, e.g. $\dot{\boldsymbol{\phi}}^{\dagger} \equiv\left(\gamma_{i j} \dot{\phi}^{j}\right)$ and $\nabla^{\dagger} \equiv$ $\left(\gamma^{i j} \nabla_{j}\right)$. Here and in the following, we use $\nabla_{j}$ to denote the covariant derivative constructed from the field-space metric $\left(\gamma_{i j}\right)$.

For homogeneous fields in flat FLRW space-time, Eqs. (31) and (32) reduce to the Friedmann equations and the equations of motion for the scalar fields,

$$
\begin{gathered}
H^{2}=\frac{1}{3 M_{P}^{2}}\left(\frac{1}{2}|\dot{\boldsymbol{\phi}}|^{2}+\tilde{U}\right), \\
2 \dot{H}+3 H^{2}=-\frac{1}{M_{P}^{2}}\left(\frac{1}{2}|\dot{\boldsymbol{\phi}}|^{2}-\tilde{U}\right), \\
\frac{D \dot{\boldsymbol{\phi}}}{d t}+3 H \dot{\boldsymbol{\phi}}=-\nabla^{\dagger} \tilde{U},
\end{gathered}
$$

where a dot stands for a derivative with respect to $t$, $H=\dot{a} / a$, and the action of $D$ on a contravariant vector 
$X^{i}$ is defined as $D X^{i}=d X^{i}+\Gamma_{a b}^{c} X^{a} d \phi^{b}$. To this we can add the equation for the current Eq. (39), which for homogeneous fields reduces to

$$
\frac{d}{d t}\left(a^{3} \dot{\boldsymbol{\phi}} \cdot \Delta \boldsymbol{\phi}\right)=4 \tilde{V}_{\Lambda_{0}},
$$

where $\Delta \boldsymbol{\phi}=\left(\Delta \phi^{1}, \Delta \phi^{2}\right)$. This relation is of course not independent of the equations of motion. However, it will prove useful in the following.

For the discussion of inflation, it is helpful to change the time parameter from $t$ to the e-fold time parameter $N=\ln a(t)$. The field equations can then be written as

$$
\begin{gathered}
H^{2}=\frac{\tilde{U}}{3 M_{P}^{2}-\frac{1}{2}\left|\boldsymbol{\phi}^{\prime}\right|^{2}}, \\
\frac{H^{\prime}}{H}=-\frac{1}{2} \frac{\left|\boldsymbol{\phi}^{\prime}\right|^{2}}{M_{P}^{2}}, \\
\frac{\frac{D \boldsymbol{\phi}^{\prime}}{d N}}{3-\frac{1}{2}\left|\boldsymbol{\phi}^{\prime}\right|^{2} / M_{P}^{2}}+\boldsymbol{\phi}^{\prime}=-M_{P}^{2} \nabla^{\dagger} \ln \tilde{U},
\end{gathered}
$$

where a prime denotes a derivative with respect to the e-fold parameter $N$.

\section{Slow-roll inflation and background trajectories}

In the present model, inflation can occur due to a phase of slow-roll of the scalar fields over the flat part of the potential towards one of the potential valleys (cf. Fig. 1). Let us define the slow-roll parameter $\epsilon$ and the slow-roll vector $\boldsymbol{\eta}$ as $[44,45]^{11}$

$$
\begin{gathered}
\epsilon \equiv-\frac{H^{\prime}}{H}=\frac{1}{2} \frac{\left|\boldsymbol{\phi}^{\prime}\right|^{2}}{M_{P}^{2}}, \\
\boldsymbol{\eta} \equiv \frac{D \boldsymbol{\phi}^{\prime}}{d N} /\left|\boldsymbol{\phi}^{\prime}\right|,
\end{gathered}
$$

in terms of which Eqs. (62) and (64) read

$$
\begin{gathered}
H^{2}=\frac{1}{M_{P}^{2}} \frac{\tilde{U}}{3-\epsilon}, \\
\frac{\boldsymbol{\eta}}{3-\epsilon}\left|\boldsymbol{\phi}^{\prime}\right|+\boldsymbol{\phi}^{\prime}=-M_{P}^{2} \nabla^{\dagger} \ln \tilde{U} .
\end{gathered}
$$

The exact condition for inflation, i.e. for $\ddot{a}>0$, is given by $\epsilon<1$.

The slow-roll regime is characterized by the fact that the Eqs. (67) and (68) are well-approximated by the "slow-roll equations"

\footnotetext{
${ }^{11}$ The definition of the vector $\boldsymbol{\eta}$ used here differs from the definition in [44] by the factor $\left|\boldsymbol{\phi}^{\prime}\right|$.
}

$$
\begin{gathered}
H^{2}=\frac{\tilde{U}}{3 M_{P}^{2}}, \\
\boldsymbol{\phi}^{\prime}=-M_{P}^{2} \nabla^{\dagger} \ln \tilde{U} .
\end{gathered}
$$

The conditions for the validity of the slow-roll approximation are $^{12}$

$$
\epsilon \ll 1 \quad \text { and } \quad \eta \equiv|\eta| \ll 1 .
$$

Still following Ref. [44], we introduce the kinematical orthonormal basis vectors $\boldsymbol{e}_{\|}=\frac{\boldsymbol{\phi}^{\prime}}{\left|\boldsymbol{\phi}^{\prime}\right|}$, pointing in the direction of the field trajectory, and $\boldsymbol{e}_{\perp}$, pointing in the direction of $\left(\boldsymbol{I}-\boldsymbol{e}_{\|} \boldsymbol{e}_{\|}^{\dagger}\right) \boldsymbol{\eta}$, where $\boldsymbol{I}$ is the $2 \times 2$ unit matrix. This allows us to write the second slow-roll parameter as

$$
\eta=\sqrt{\eta_{\|}^{2}+\eta_{\perp}^{2}}
$$

where the speed-up rate $\eta_{\|}$and the turn-rate $\eta_{\perp}$ are defined as

$$
\begin{gathered}
\eta_{\|}=\boldsymbol{e}_{\|} \cdot \boldsymbol{\eta}=-\frac{3-\epsilon}{\left|\boldsymbol{\phi}^{\prime}\right|}\left(\left|\boldsymbol{\phi}^{\prime}\right|+M_{P}^{2} \boldsymbol{e}_{\|} \cdot \boldsymbol{\nabla} \ln \tilde{U}\right), \\
\eta_{\perp}=\boldsymbol{e}_{\perp} \cdot \boldsymbol{\eta}=-M_{P}^{2} \frac{3-\epsilon}{\left|\boldsymbol{\phi}^{\prime}\right|} \boldsymbol{e}_{\perp} \cdot \nabla \ln \tilde{U} .
\end{gathered}
$$

In the case of one-field inflation, $\eta_{\perp}$ is equal to zero, and $\eta=\left|\eta_{\|}\right|$. In the upcoming section, we will see that for the Higgs-dilaton model, as long as the fields are in the scaleinvariant region, $\eta_{\perp}$ goes to zero very quickly, and $\eta$ can be computed like in a single-field model.

Within the slow-roll approximation, i.e. making use of Eqs. (69) and (70), one can compute approximations in terms of the potential for the slow-roll parameters $\epsilon$ and $\eta$

$$
\begin{gathered}
\epsilon^{(\mathrm{SR})}=\frac{1}{2} M_{P}^{2}|\nabla \ln \tilde{U}|^{2}, \\
\eta^{(\mathrm{SR})}=\sqrt{\left(\boldsymbol{e}_{\|}^{(\mathrm{SR})}\right)^{\dagger} \boldsymbol{M}^{2} \boldsymbol{e}_{\|}^{(\mathrm{SR})},}
\end{gathered}
$$

such as for the speed-up rate $\eta_{\|}$and the turn rate $\eta_{\perp}$

$$
\begin{aligned}
& \eta_{\|}^{(\mathrm{SR})}=-\left(\boldsymbol{e}_{\|}^{(\mathrm{SR})}\right)^{\dagger} \boldsymbol{M} \boldsymbol{e}_{\|}^{(\mathrm{SR})}, \\
& \eta_{\perp}^{(\mathrm{SR})}=-\left(\boldsymbol{e}_{\|}^{(\mathrm{SR})}\right)^{\dagger} \boldsymbol{M} \boldsymbol{e}_{\perp}^{(\mathrm{SR})},
\end{aligned}
$$

where the matrix $\boldsymbol{M}$ is defined as

$$
\boldsymbol{M} \equiv M_{P}^{2} \nabla^{\dagger} \nabla \ln \tilde{U}
$$

and the kinematical unit vectors in the slow-roll approximation are given by

\footnotetext{
${ }^{12}$ Note that $\epsilon=\epsilon_{1}$ and $|\eta|=\frac{1}{2}\left|\epsilon_{2}\right|$, where $\epsilon_{1} \equiv \frac{H^{\prime}}{H}$ and $\epsilon_{2} \equiv$ $\frac{D \ln \epsilon}{d N}$ are the multifield generalizations of the standard first two horizon-flow parameters defined in [46].
} 


$$
\begin{aligned}
& \boldsymbol{e}_{\|}^{(\mathrm{SR})}=-\frac{\boldsymbol{\nabla}^{\dagger} \ln \tilde{U}}{|\boldsymbol{\nabla} \ln \tilde{U}|}, \quad \text { and } \\
& \boldsymbol{e}_{\perp}^{(\mathrm{SR})}=-\frac{\boldsymbol{M}+\eta_{\|}^{(\mathrm{SR})} \boldsymbol{I}}{\sqrt{\left(\eta^{(\mathrm{SR})}\right)^{2}-\left(\eta_{\|}^{(\mathrm{SR})}\right)^{2}}} \boldsymbol{e}_{\|}^{(\mathrm{SR})} .
\end{aligned}
$$

Hence, instead of the exact slow-roll conditions (71), one can use the approximate slow-roll conditions

$$
\epsilon^{(\mathrm{SR})} \ll 1 \text { and } \eta^{(\mathrm{SR})} \ll 1,
$$

which should be understood as consistency conditions for the slow-roll approximation. Once the system is in the slow-roll regime, i.e. the exact conditions (71) are satisfied, the approximate conditions (78) guarantee that the system remains in the slow-roll regime and describes a phase of inflation. We will approximate the time where inflation ends as the moment where $\epsilon^{(\mathrm{SR})}=1$.

Let us now discuss the regions in the $(\chi, h)$-plane for which the approximate slow-roll conditions hold (cf. Fig. 2). The slow-roll region extends to infinity along the potential valleys if $\xi_{\chi}<\frac{1}{2}$. As will be shown in Sec. IV, only if this condition holds, the scalar fields can constitute a dark energy component in the late stage. Further, during inflation, it is safe to neglect the term in the potential proportional to $\alpha$. In fact, for $\alpha=0$, the potential possesses only one valley which goes along the $\chi$-axis. For $\alpha \neq 0$, this valley splits into two valleys that lie at the angles $\theta= \pm \arctan (\alpha)$ with respect to the $\chi$-axis. For $\alpha \lll 1$, these angles are very small. We will see that inflation in our model occurs far from these valleys where the effect of a nonzero $\alpha$ is irrelevant. Hence, we will put $\alpha=0$ for the rest of this section. The plot of the slow-roll region for $\xi_{\chi}<\frac{1}{2}$ and $\alpha=0$ is presented in Fig. 2.

Next, we want to analyze the different trajectories the fields can take if the initial conditions are chosen in the slow-roll region. We will only consider trajectories starting in the first quadrant $\chi, h>0$. Trajectories starting in other quadrants are exactly analog. The shape of the potential (29) makes that all trajectories tend to approach one of the potential valleys. There are trajectories (type a) that on their way to the valley never leave the slow-roll region. Numerical computations show that such trajectories undergo only very few slow oscillations before asymptotically approaching the valley. One can not expect a successful reheating period from this type of behavior $[31,32]$. The good trajectories (type b) are those that at some point leave the slow-roll region. After the exit of the slow-roll region, which, at the same time, marks the end of inflation, these trajectories undergo a fast roll towards the valley and, therefore, oscillate strongly around its minimum. Typical examples for both types of trajectories are given in Fig. 2.

Looking at Fig. 2, we observe that all good trajectories (type b, blue line) go through the scale-invariant region before leaving the slow-roll region. Therefore, for these trajectories, the end of inflation always takes place within

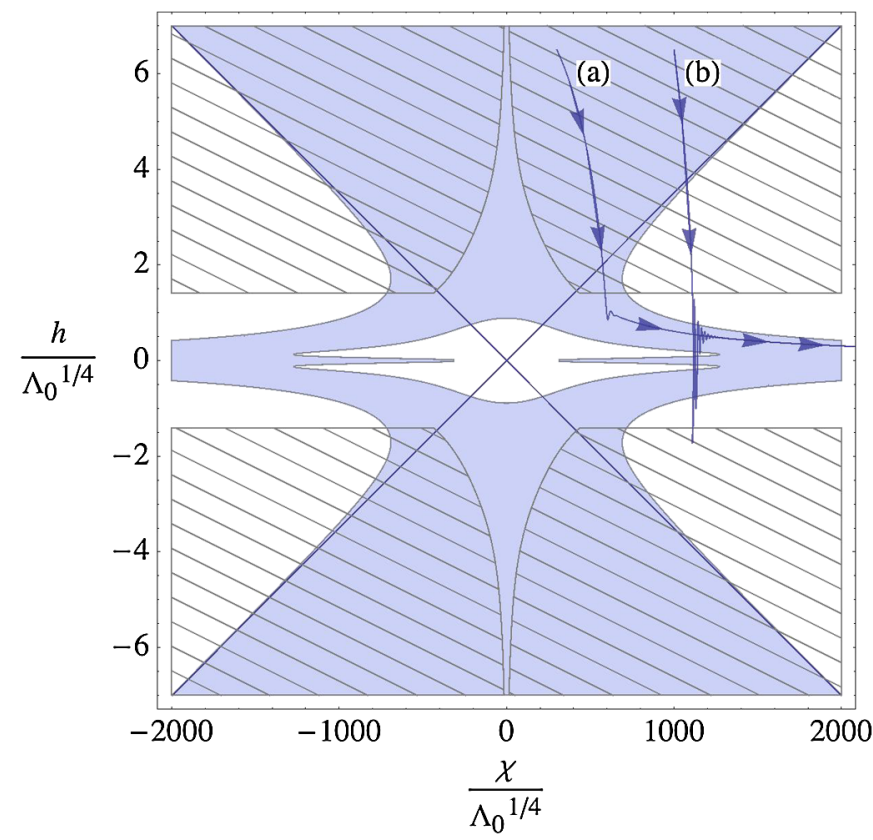

FIG. 2 (color online). The blue (shaded) region is the slow-roll region for $\xi_{\chi} \ll 1, \xi_{h} \gg 1$, and $\alpha=0$, given by $\epsilon^{(\mathrm{SR})}<1$. The inclusion of the second slow-roll condition $\eta^{(\mathrm{SR})}<1$ does not change the essential properties of this region. The general features of the slow-roll region are the same whenever $\xi_{\chi}<\frac{1}{2}$ and $\xi_{h}>\frac{1}{2}$. For $\xi_{\chi}<\frac{1}{2}$ and $\xi_{h}<\frac{1}{2}$, the central fast-rolling region vanishes. For $\xi_{\chi}>\frac{1}{2}$, the slow-roll region does not extend to infinity along the $\chi$-axis, in which case the scalar fields cannot act as dark energy in the late stage of evolution. The dashed region corresponds to the scale-invariant region delimited by $v_{1}<1$ and $v_{2}<1$. This is the region where the influence of $\Lambda_{0} \neq 0$ is small. The presence of the slow-roll region along the $\chi$-axis, such as the central fast-roll region, are effects of $\Lambda_{0}>0$. For $\Lambda_{0}=0$, the slow-roll region is simply given by the triangles delimited by the two diagonal lines. Note that, in this case, the units of the axis have to be chosen differently. The curves with arrows represent typical trajectories of the background fields. Trajectories of type (a) never leave the slow-roll region. Trajectories of type (b) leave the slow-roll region and oscillate strongly before rolling down the valley. These trajectories were found by numerically solving the exact equations (60).

the scale-invariant region. We will see in Sec. IV B that requiring the scalar fields to act as a dark-energy component in the late phase will give a bound on the initial conditions. Qualitatively, this bound tells that the scalar fields during inflation have to be very far from the origin. Therefore, not only the end, but the whole period of observable inflation (i.e. the final $\sim 60$ e-folds) takes place in the scale-invariant region. This fact considerably simplifies the analysis. In particular, during inflation the scaleinvariance-breaking part $\tilde{V}_{\Lambda_{0}}$ of the potential (29) can be neglected, i.e. $\tilde{U} \simeq \tilde{V}$. As a consequence, if one uses the set of variables $(\rho, \theta)$ introduced in Sec. III A 2, the potential only depends on $\theta$. Consequently, also the slow-roll parameters $\epsilon^{(\mathrm{SR})}$ and $\eta^{(\mathrm{SR})}$ are functions of $\theta$ only. Further, 
one finds that the lowest order approximation of the turn rate $\eta_{\perp}$ vanishes, i.e. $\eta_{\perp}^{(\mathrm{SR})}=0$.

In the scale-invariant region and written in terms of the variables $(\rho, \theta)$, the slow-roll equations for the scalar fields (70) read

$$
\begin{gathered}
\rho^{\prime}=0, \\
\theta^{\prime}=-\frac{4 \xi_{\chi}}{1+6 \xi_{\chi}} \cot \theta\left(1+\frac{6 \xi_{\chi} \xi_{h}}{\xi_{\chi} \cos ^{2} \theta+\xi_{h} \sin ^{2} \theta}\right) .
\end{gathered}
$$

From the first equation, one finds that the background trajectories in the scale-invariant slow-roll region correspond to a constant value for $\rho, \rho=\rho_{0}$, respectively, to ellipses in the $(\chi, h)$-plane described by

$$
\left(1+6 \xi_{\chi}\right) \chi^{2}+\left(1+6 \xi_{h}\right) h^{2}=M_{P}^{2} e^{2 \rho_{0} / M_{P}} .
$$

The second scalar field equation (83), as a consequence of scale invariance, does not depend on $\rho_{0}$. This equation can be integrated in order to get the number of e-folds before the end of inflation as a function of the angle $\theta$,

$$
\begin{aligned}
N\left(\theta, \theta_{\text {end }}\right)= & \frac{1}{4 \xi_{\chi}}\left[\ln \left(\frac{\cos \theta_{\text {end }}}{\cos \theta}\right)\right. \\
& \left.+3 \xi_{\chi} \ln \left(\frac{\xi_{\chi} \cos ^{2} \theta_{\text {end }}+\xi_{h} \sin ^{2} \theta_{\text {end }}+6 \xi_{\chi} \xi_{h}}{\xi_{\chi} \cos ^{2} \theta+\xi_{h} \sin ^{2} \theta+6 \xi_{\chi} \xi_{h}}\right)\right],
\end{aligned}
$$

where $\theta_{\text {end }}$ is the value of $\theta$ at the end of inflation, which can be found from the condition

$\epsilon^{(\mathrm{SR})}\left(\theta_{\text {end }}\right)=\frac{8 \xi_{\chi}^{2}\left(1+6 \xi_{h}\right)}{1+6 \xi_{\chi}} \frac{\cot ^{2} \theta_{\text {end }}}{\xi_{\chi} \cos ^{2} \theta_{\text {end }}+\xi_{h} \sin ^{2} \theta_{\text {end }}}=1$.

After inserting values for $\xi_{\chi}, \xi_{h}$, and requiring a minimal number of inflationary e-folds $N\left(\theta_{\min }, \theta_{\text {end }}\right)=N_{\min }$, Eqs. (85) and (86) can be solved to obtain a lower bound $\theta_{\text {min }}<\theta_{\text {initial }}$ on the initial conditions for inflation. In what follows, we will derive bounds on the parameters $\xi_{\chi}$ and $\xi_{h}$, which are related to the spectra of primordial perturbations.

\section{One-field attractor in the scale-invariant region}

In the previous section, we have seen that if the slow-roll conditions hold and if the system is in the scale-invariant region, i.e. $v_{1} \ll 1$ and $v_{2} \ll 1$, the trajectories are given by $\rho=\rho_{0}$. In other words, there exists a set of variables in term of which only one of the two fields evolves during inflation.

In this section, we are going to show that in the scaleinvariant region, $\rho^{\prime}=0$ is an attractor independently of slow-roll and also after inflation. We will further show that during inflation in the scale-invariant region, the turn-rate $\eta_{\perp}$ goes to zero very rapidly, also beyond the lowest order slow-roll approximation. This fact will have important consequences for the evolution of perturbations of the scalar fields.

Let us look at the equation for the scale current (61). In the scale-invariant region, the term on the right-hand side can be neglected. In terms of the variables $(\rho, \theta)$ and using the e-fold time parameter, one obtains

$$
\rho^{\prime}=\frac{\text { cst. }}{H \gamma_{\rho \rho}} e^{-3 N},
$$

where the constant depends on initial conditions. $\gamma_{\rho \rho}$ can be read from Eq. (44) and represents a bounded function. During inflation, the factor $H^{-1}$ is generally nearly constant but grows at most like $e^{N}$ at the end of inflation. For a matter-domination-like stage, one obtains $H^{-1} \propto e^{1 / 2 N}$, and for a radiation-domination-like stage $H^{-1} \propto e^{2 / 3 N}$. One can read from Eqs. (62) and (63) that whenever the potential is positive, which is always the case in the scaleinvariant region of our model, $H^{-1}$ grows more slowly than $e^{3 N}$. We conclude that as soon as the system can be approximated by the scale-invariant equations, $\rho^{\prime}=0$ is an attractor.

Let us now turn our attention to the turn rate $\eta_{\perp}$. In the scale-invariant region, Eq. (71) reduces to

$$
\begin{aligned}
\eta_{\perp} & =-M_{P}^{2} \frac{3-\epsilon}{\left|\boldsymbol{\phi}^{\prime}\right|} \boldsymbol{e}_{\perp} \cdot \nabla \ln \tilde{V} \\
& =-M_{P}^{2}|\nabla \ln \tilde{V}| \frac{3-\epsilon}{\left|\boldsymbol{\phi}^{\prime}\right|} \boldsymbol{e}_{\perp} \cdot \boldsymbol{e}_{(\nabla \tilde{V})},
\end{aligned}
$$

where $\boldsymbol{e}_{(\nabla \tilde{V})}$ is a unit vector pointing in the direction of $\boldsymbol{\nabla} \tilde{V}$. From the current conservation law (61), still neglecting $\tilde{V}_{\Lambda_{0}}$ and noticing that $\Delta \boldsymbol{\phi}$ is perpendicular to $\boldsymbol{\nabla} \tilde{V}$, we find

$$
\boldsymbol{e}_{\|} \cdot \boldsymbol{e}_{(\nabla \tilde{V})_{\perp}}=\frac{\text { cst. }}{\left|\Delta \boldsymbol{\phi} \| \boldsymbol{\phi}^{\prime}\right| H} e^{-3 N},
$$

where $\boldsymbol{e}_{(\nabla \tilde{V}) \perp}$ is a unit vector perpendicular to $\boldsymbol{e}_{(\nabla \tilde{V})}$. At every point in field space, one can find an orthogonal matrix $\boldsymbol{O}$ such that $\boldsymbol{e}_{\perp}=\boldsymbol{O} \boldsymbol{e}_{\|}$and $\boldsymbol{e}_{(\nabla \tilde{V})}=\boldsymbol{O}_{(\nabla \tilde{V})_{\perp}}$. Hence, one has

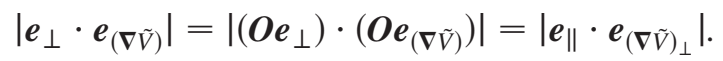

Inserting this relation and Eq. (89) in Eq. (88) and making use of Eqs. (65) and (75), we find the following result for the turn rate:

$$
\left|\eta_{\perp}\right|=\operatorname{cst} . \cdot \frac{(3-\epsilon) \sqrt{\epsilon^{(\mathrm{SR})}}}{\epsilon H|\Delta \boldsymbol{\phi}|} e^{-3 N}
$$

where we have redefined the constant related to initial conditions. During slow-roll inflation, $\epsilon$ is growing, but 
remains smaller than unity, $H$ is decreasing very slowly, and $\epsilon^{(\mathrm{SR})}$ is small. $|\Delta \boldsymbol{\phi}|$ is a bounded function, which in terms of the variables $(\rho, \theta)$, is given by $|\Delta \boldsymbol{\phi}|=M_{P} \sqrt{\gamma_{\rho \rho}}$. We conclude that during slow-roll inflation and for approximate scale invariance, the turn rate $\eta_{\perp}$ goes to zero exponentially fast. Further, one can show that if scale invariance is slightly violated, $\eta_{\perp}$ is proportional to $v_{2}$.

\section{Linear perturbations}

The theory of cosmological perturbations as stemming from quantum fluctuations during inflation was developed in [47-51] (see also [52] and references therein). Including scalar and tensor perturbations and fixing the Newtonian transverse traceless gauge, the line element can be written as

$$
d s^{2}=-(1+2 \Phi) d t^{2}+a(t)^{2}\left((1-2 \Psi) \delta_{i j}+h_{i j}^{T T}\right) d x^{i} d x^{j}
$$

$\Phi$ and $\Psi$ are the Bardeen potentials [53]. For comparison with observations of the Cosmic Microwave Background (CMB), we will be interested in the power spectrum of the comoving curvature perturbation, which is defined as $[53,54]$

$$
\zeta \equiv \Psi-\frac{H}{\dot{H}}(\dot{\Psi}+H \Phi) .
$$

Through the perturbed Einstein equations, $\zeta$ is related to the linear perturbations of the scalar fields, $\boldsymbol{\delta} \boldsymbol{\phi}$, like (see, e.g. [55])

$$
\zeta=\frac{1}{\left|\boldsymbol{\phi}^{\prime}\right|}\left(\Psi \boldsymbol{\phi}^{\prime}+\boldsymbol{\delta} \boldsymbol{\phi}\right) \cdot \boldsymbol{e}_{\|}
$$

where the quantity in parenthesis is the multifield version of the Mukhanov-Sasaki variable [56,57]. The evolution equation for $\zeta$ is given by [42-44]

$$
\zeta^{\prime}=\frac{2}{(a H)^{2}\left|\boldsymbol{\phi}^{\prime}\right|^{2}} \Delta \Psi-2 \eta_{\perp} \frac{\delta \phi_{\perp}}{\left|\boldsymbol{\phi}^{\prime}\right|},
$$

where $\Delta=\delta^{i j} \partial_{i} \partial_{j}$. The quantity $\delta \phi_{\perp} \equiv \boldsymbol{\delta} \boldsymbol{\phi} \cdot \boldsymbol{e}_{\perp}$ is the component of the field perturbations perpendicular to the background field trajectory sometimes called relative isocurvature (or entropy) perturbation (see, e.g. [58]). In the long wavelength limit, $k \ll a H$, the first term in Eq. (95) can be neglected, and the evolution equation becomes

$$
\zeta^{\prime}=-2 \eta_{\perp} \frac{\delta \phi_{\perp}}{\left|\phi^{\prime}\right|}
$$

This is a well-known result, showing that for multifield inflation, $\zeta$ is not in general conserved outside the
Hubble horizon $[42,43]$. There are two cases in which the source term on the right-hand side of the equation vanishes. One is if the perturbation vector $\boldsymbol{\delta} \boldsymbol{\phi}$ is tangent to the field trajectory, i.e. $\delta \phi_{\perp}=0$. This corresponds to the complete absence of relative isocurvature perturbations during inflation and is not satisfied in our scenario. The second possibility is to have the potential gradient $\nabla U$ parallel to the background field trajectory, resulting in the vanishing of the turn rate $\eta_{\perp}=0$. In our model, as we have seen in the previous sections, inflation takes place in the scale-invariant region, where $\eta_{\perp}=0$ is an attractor. Hence, as a consequence of scale invariance, the comoving curvature perturbation is practically conserved outside the horizon, just like in singlefield inflation. ${ }^{13}$ As mentioned before, corrections due to deviations from scale invariance are suppressed by the parameter $\boldsymbol{v}_{2}$. This parameter being extremely small (cf. Sec. IV B), we will neglect such corrections.

We will from now on use the fact that inflation takes place in the scale-invariant region and suppose that initial conditions are such that the attractor $\eta_{\perp}=0$ has been reached before the observable scales cross the horizon. In that case, $\zeta$ is constant outside the Hubble horizon, and the result for the primordial spectrum of $\zeta$ is like in the case of one-field inflation. To lowest order in the slow-roll parameters, it can be expressed as $[52,59,60]$

$$
\mathcal{P}_{\zeta}(k) \simeq \frac{1}{2 M_{P}^{2} \epsilon^{*}}\left(\frac{H^{*}}{2 \pi}\right)^{2} \simeq \frac{1}{24 \pi^{2} \epsilon^{(\mathrm{SR}) *}} \frac{\tilde{V}^{*}}{M_{P}^{4}},
$$

where quantities with an asterisk are evaluated at the moment of horizon crossing, i.e. when $a H=k$. The scalar spectral index is given by

$$
\begin{aligned}
n_{s}(k)-1 & \equiv \frac{d \ln \mathcal{P}_{\zeta}}{d \ln k} \simeq-2\left(\epsilon^{*}+\eta_{\|}^{*}\right) \\
& \simeq-2\left(\epsilon^{(\mathrm{SR}) *}+\eta_{\|}^{(\mathrm{SR}) *}\right),
\end{aligned}
$$

while the running of the spectral index can be expressed as [61]

$$
\begin{aligned}
\alpha_{\zeta}(k) & \equiv \frac{d n_{s}}{d \ln k} \simeq-4 \epsilon^{*} \eta_{\|}^{*}-2 \eta_{\|}^{*} \xi^{*} \\
& \simeq-4 \epsilon^{(\mathrm{SR}) *} \eta_{\|}^{(\mathrm{SR}) *}-2\left(\eta_{\|}^{*} \xi^{*}\right)^{(\mathrm{SR})},
\end{aligned}
$$

where $\xi$ is the third Hubble-flow parameter $\xi \equiv \frac{d^{2} \ln \epsilon}{d N^{2}}$. In the slow-roll approximation, the combination $\eta_{\|} \xi$ can be expressed in terms of the potential as [44]

$$
\left(\eta_{\|} \xi\right)^{(\mathrm{SR})}=\boldsymbol{e}_{\|}^{(\mathrm{SR}) \dagger} \boldsymbol{X} \boldsymbol{e}_{\|}^{(\mathrm{SR})}
$$

\footnotetext{
${ }^{13}$ Using the results of [44], one finds that $\delta \phi_{\perp} /\left|\boldsymbol{\phi}^{\prime}\right|$ is of the same order of magnitude as $\zeta$ and does not grow considerably during inflation.
} 
with the matrix $\boldsymbol{X} \equiv M_{P}^{2} \nabla \ln \tilde{U} \nabla^{\dagger} \boldsymbol{M}$, where $\tilde{U}$ can be replaced by $\tilde{V}$ due to scale invariance. ${ }^{14}$

Now, even if the relative entropy perturbations $\delta \phi_{\perp}$ do not affect the evolution of $\zeta$, since they are completely decoupled, they can in general be present at the end of inflation. The total entropy perturbations, however, are given by $\mathcal{S} \propto \eta_{\perp} \delta \phi_{\perp} /\left|\boldsymbol{\phi}^{\prime}\right|$ [58]. And, as we have shown above, scale invariance during inflation leads to $\eta_{\perp} \simeq 0$ and, hence, to a strong suppression of $\mathcal{S}$. As has been shown in Ref. [62] (see also [63] for a general perspective), the large scale suppression of entropy perturbations during inflation avoids the resonant growth of these fluctuations also during (p)reheating. Working with this assumption, we will be able to relate the primordial spectra to $\mathrm{CMB}$ observations.

The primordial spectrum of the tensor perturbations is given, to the lowest order in the slow-roll approximation, by $[52,59]^{15}$

$$
\mathcal{P}_{g}(k) \simeq \frac{8}{M_{P}^{2}}\left(\frac{H^{*}}{2 \pi}\right)^{2} \simeq \frac{2}{3 \pi^{2}} \frac{\tilde{V}^{*}}{M_{P}^{4}},
$$

which results in a tensorial spectral index:

$$
n_{g}(k) \equiv \frac{d \ln \mathcal{P}_{g}}{d \ln k} \simeq-2 \epsilon^{*} \simeq-2 \epsilon^{(\mathrm{SR}) *} .
$$

The ratio of the tensor and the scalar spectra to first order in slow-roll is then given by

$$
r \equiv \frac{\mathcal{P}_{g}}{\mathcal{P}_{\zeta}} \simeq 16 \epsilon^{*} \simeq 16 \epsilon^{(\mathrm{SR}) *},
$$

and we have the consistency condition like in one-field inflation

$$
r=-8 n_{g}
$$

valid to the lowest nontrivial order in the slow-roll approximation.

\footnotetext{
${ }^{14}$ Let us mention again that, since inflation takes place in the scale-invariant region, one can choose variables $(\rho, \theta)$ for which the approximate slow-roll parameters $\epsilon^{(\mathrm{SR})}, \eta_{\|}^{(\mathrm{SR})}$ and the combination $\left(\eta_{\|} \xi\right)^{(\mathrm{SR})}$ depend only on $\theta$. In models of one-field inflation with a canonical kinetic term, an alternative common definition of approximate slow-roll parameters is given by $\epsilon_{s} \equiv$ $\frac{1}{2} M_{P}^{2}\left(\frac{U^{\prime}}{U}\right)^{2}, \eta_{s} \equiv M_{P}^{2} \frac{U^{\prime \prime}}{U}$, and $\xi_{s} \equiv M_{P}^{4} \frac{U^{\prime \prime \prime} U^{\prime}}{U^{2}}$. In the case of one or several fields with noncanonical kinetic terms, these definitions generalize to $\epsilon_{s} \equiv \frac{1}{2} M_{P}^{2}|\nabla \ln U|^{2}, \quad \eta_{s} \equiv\left(\boldsymbol{e}_{\|}^{(\mathrm{SR})}\right)^{\dagger} \boldsymbol{M}_{s} \boldsymbol{e}_{\|}^{(\mathrm{SR})}$, and $\xi_{s} \equiv\left(\boldsymbol{e}_{\|}^{(\mathrm{SR})}\right)^{\dagger} \boldsymbol{X}_{s} \boldsymbol{e}_{\|}^{(\mathrm{SR})}$, where the matrices $\boldsymbol{M}_{s}$ and $\boldsymbol{X}_{s}$ are defined as $\boldsymbol{M}_{s} \equiv M_{P}^{2} \frac{1}{U} \boldsymbol{\nabla}^{\dagger} \boldsymbol{\nabla} U$ and $\boldsymbol{X}_{s} \equiv M_{P}^{4} \frac{1}{U^{2}} \boldsymbol{\nabla} U \boldsymbol{\nabla}^{\dagger} \boldsymbol{\nabla}^{\dagger} \boldsymbol{\nabla} U$, and the unit vectors are given in Eq. (80). These parameters are related to the approximate slow-roll parameters used in the present work as $\epsilon^{(\mathrm{SR})}=\epsilon_{s}, \eta_{\|}^{(\mathrm{SR})}=2 \epsilon_{s}-\eta_{s}$ and $\left(\eta_{\|} \xi\right)^{(\mathrm{SR})}=8 \epsilon_{s}^{2}-$ $6 \epsilon_{s} \eta_{s}+\xi_{s}$.

${ }^{15}$ This result is based on the slow-roll approximation and involves no further assumptions.
}

\section{CMB constraints on parameters and predictions of the model}

In this section, we are going to explicitly compute the primordial spectra and confront them with CMB observations. As discussed in the previous sections, the whole period of observable inflation takes place in the scaleinvariant region. We can therefore use Eqs. (85) and (86) for the background. Moreover, due to scale invariance, $\zeta$ is conserved for large wavelengths during inflation. Further, we make the assumption that after inflation, entropy perturbations die away before having an observable effect. This allows us to directly compare the primordial spectra (97) and (100) to observations of the CMB.

We will show in the following that the running of the scalar spectral index and the amplitude of tensor perturbations are related and very small, cf. Eq. (119). Therefore, we can consider those observational bounds (WMAP7 + $\mathrm{BAO}+\mathrm{H}_{0}$ ) for the scalar tilt and the amplitude of the scalar power spectrum, which are based on the standard $\Lambda \mathrm{CDM}$ model and the assumptions that the primordial spectrum obeys a power-law and that tensor modes can be neglected (see Ref. [64]):

$$
\begin{gathered}
\mathcal{P}_{\zeta}\left(k_{0}\right)=(2.43 \pm 0.27) \times 10^{-9}, \\
n_{s}\left(k_{0}\right)=0.968 \pm 0.036,
\end{gathered}
$$

where $k_{0} / a_{0}=0.002 \mathrm{Mpc}^{-1}$, and the indicated errors correspond to the $99 \%$ confidence levels.

Let us start by computing the spectral quantities $\mathcal{P}_{\zeta}\left(k_{0}\right)$, $n_{s}\left(k_{0}\right), \alpha\left(k_{0}\right)$ and $r\left(k_{0}\right)$, evaluated at the pivot scale $k_{0}$, in terms of the parameters $\xi_{\chi}, \xi_{h}$, and $\lambda$. This is done in four steps:

(i) Equation (86) is solved for $\theta_{\text {end }}=\theta_{\text {end }}\left(\xi_{\chi}, \xi_{h}\right)$.

(ii) $\theta_{\text {end }}$ is inserted into Eq. (85), from which one determines $\theta^{*}=\theta^{*}\left(\xi_{\chi}, \xi_{h}, N^{*}\right)$.

(iii) Expressions (97)-(99) and (103) are evaluated at $\theta^{*}$ to find the spectral quantities as functions of $\xi_{\chi}, \xi_{h}$, $\lambda$, and $N^{*}$.

(iv) $N^{*}$, the number of e-folds between the moment where $k_{0}$ exits the horizon and the end of inflation, is expressed as a function of the parameters $\xi_{\chi}, \xi_{h}$, and $\lambda$.

In order to determine $N^{*}$ (step (iv)) we need to know the post-inflationary evolution of the Universe, including the details of the reheating process. If there are uncertainties related to the post-inflationary history, these can be accounted for by varying the value of $N^{*}$. One can compute $N^{*}$ approximately by making a few assumptions about the post-inflationary evolution. First, during the reheating phase, the scale factor is expected to evolve like in a matter-dominated universe. The reason is that, during this stage, the present model behaves much like the HiggsInflation model (cf. [31,32]). In this sense, matterlike scaling of the Universe during reheating is not really an 
assumption, but rather a property of the considered model. Next, we make the usual assumptions that reheating is followed by the standard radiation- and matter-dominated stages. Further assuming that the transitions between the different phases are instantaneous, one can derive the following relation (cf. [59])

$$
\begin{aligned}
N^{*} \simeq & -\ln \frac{k_{0}}{a_{0} H_{0}}-\ln \left(\frac{\varrho_{0}^{c r} / \Omega_{0}^{\gamma}}{\tilde{V}\left(\theta^{*}\right)}\right)^{1 / 4}+\ln \left(\frac{\tilde{V}\left(\theta^{*}\right)}{\tilde{V}\left(\theta_{\text {end }}\right)}\right)^{1 / 4} \\
& -\frac{1}{3} \ln \left(\frac{\tilde{V}\left(\theta_{\text {end }}\right)}{\varrho_{r h}}\right)^{1 / 4} .
\end{aligned}
$$

Here, $a_{0}, H_{0}, \varrho_{0}^{c r}$, and $\Omega_{0}^{r}$ stand for the current values of the scale factor, the Hubble parameter, the critical density, and the abundance of radiation, respectively. $\varrho_{r h}$ denotes the radiation energy density at the end of reheating, i.e. at the onset of the hot big bang. After inserting the observational value $\Omega_{0}^{r} h^{2} \simeq 4.2 \times 10^{-5}$ (for $T_{0}^{\gamma} \simeq 2.73 \mathrm{~K}$ [65]), where $h$ is the dimensionless Hubble parameter, the above formula can be written as

$$
\begin{aligned}
N^{*} \simeq & 59-\ln \frac{k_{0} \mathrm{Mpc}}{0.002 a_{0}}-\ln \frac{10^{16} \mathrm{GeV}}{\tilde{V}\left(\theta^{*}\right)^{1 / 4}}+\ln \left(\frac{\tilde{V}\left(\theta^{*}\right)}{\tilde{V}\left(\theta_{\text {end }}\right)}\right)^{1 / 4} \\
& -\frac{1}{3} \ln \left(\frac{\tilde{V}\left(\theta_{\text {end }}\right)}{\varrho_{r h}}\right)^{1 / 4} .
\end{aligned}
$$

Notice that the dependence on $H_{0}$ has cancelled out. ${ }^{16} \mathrm{~A}$ detailed determination of $\varrho_{r h}$ goes beyond the scope of this work and is postponed for a future publication. We can, however, consider two limiting cases. An upper limit on $\varrho_{r h}$ is simply given by

$$
\varrho_{r h}^{\max }=\tilde{V}\left(\theta_{\text {end }}\right),
$$

corresponding to instantaneous reheating at the end of inflation. A lower limit can be found in the same way as for the Higgs-Inflation model in [31,32]. Namely, one can look for the value of $\theta$, below which the particle interactions become those of the standard model, apart from the suppressed interactions discussed in Sec. II A 1, and therefore guarantee immediate reheating. Inspecting the kinetic term (44) and the potential (45), we find that this happens as soon as $\tan ^{2} \theta<\min (\mu, 1) .{ }^{17}$ We will therefore set the lower limit

$$
\varrho_{r h}^{\min }=\tilde{V}\left(\theta_{r h}^{\min }\right), \quad \text { with } \quad \tan ^{2} \theta_{r h}^{\min }=\min (\mu, 1) .
$$

The value of $\lambda$ to be used when computing the spectral parameters corresponds to the Higgs self-coupling evaluated at the scale of inflation [31,32]. It contains the uncertainty related to the Higgs mass $m_{H}^{2}$. We expect the running of $\lambda$ to be similar, as in the case of the HiggsInflation model [66-73]. As was shown in [31,32], for

\footnotetext{
${ }^{16}$ In the analog formula of [59], this fact remains somewhat hidden.

${ }^{17}$ Note that the value of $s$ lies always between 1 and the value of $\mu$.
}

$m_{H}^{2} \simeq 130-180 \mathrm{GeV}$, the coupling $\lambda$ evaluated at the scale of inflation lies in the range $\lambda \simeq 0.1-1$.

Step (ii), i.e. the exact inversion of Eq. (85), can not be done analytically. Hence, before computing approximate analytical results, we execute the four steps numerically. This allows us to plot the region in parameter space, for which $\mathcal{P}_{\zeta}\left(k_{0}\right)$ and $n_{s}\left(k_{0}\right)$ lie within the observational constraints (104) and (105), cf. Fig. 3. It turns out that in the observationally allowed range and for $\lambda \simeq 0.1-1$, the spectral quantites depend on $\xi_{h}$ and $\lambda$ almost only through the combination $\xi_{h} / \sqrt{\lambda}$. This fact will become explicit in the approximate analytical results to be derived below. The red (upper) region in Fig. 3 is obtained under the assumption of instantaneous reheating $\left(\varrho_{r h}=\varrho_{r h}^{\max }\right)$, while the blue (lower) region corresponds to the case of long reheating, i.e. $\varrho_{r h}=\varrho_{r h}^{\min }$. We obtain the bounds

$$
\begin{aligned}
& 0<\xi_{\chi} \lesssim 0.008, \text { for } \varrho_{r h}=\varrho_{r h}^{\min }, \\
& 43000 \leqq \frac{\xi_{h}}{\sqrt{\lambda}} \lesssim 85000,
\end{aligned}
$$

and

$$
\begin{aligned}
& 0<\xi_{\chi} \lesssim 0.008, \text { for } \varrho_{r h}=\varrho_{r h}^{\max } . \\
& 44500 \leqq \frac{\xi_{h}}{\sqrt{\lambda}} \lesssim 92000,
\end{aligned}
$$

In this region of parameter space, the quantities $n_{s}\left(k_{0}\right), \alpha\left(k_{0}\right)$, and $r\left(k_{0}\right)$ vary only with $\xi_{\chi}$. The numerical results for these quantities are given in Figs. 4 and 5, respectively.

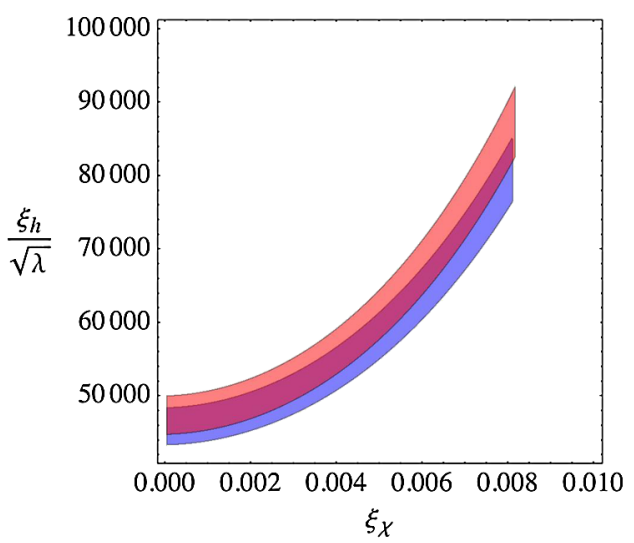

FIG. 3 (color online). This plot shows the parameter regions for which the amplitude $P_{\zeta}\left(k_{0}\right)$ and the tilt $n_{s}\left(k_{0}\right)$ of the scalar spectrum lie in the observationally allowed region (WMAP7 + $\mathrm{BAO}+H_{0}$ at $99 \%$ confidence level), for $\lambda=1$. (The variation of the result induced by a variation of $\lambda$ in the interval $0.1<$ $\lambda<1$ is negligible.) The red (upper) region is obtained for $\varrho_{r h}=\varrho_{r h}^{\max }$ (instantaneous reheating), while the blue (lower) region corresponds to $\varrho_{r h}=\varrho_{r h}^{\min }$ (long reheating). The fact that the bands are cut on the right comes from the constraint on the scalar tilt $n_{s}\left(k_{0}\right)$, cf. Eq. (116), while the band-shape is due to the constraint on the amplitude $P_{\zeta}\left(k_{0}\right)$, cf. Eq. (115). 


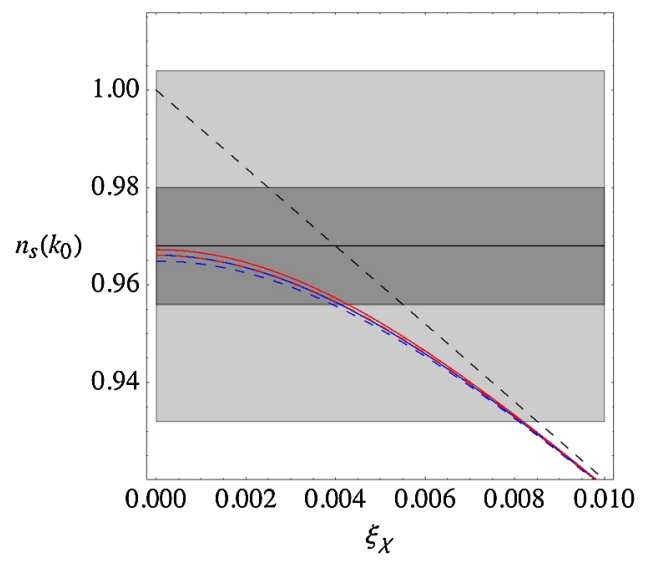

FIG. 4 (color online). The spectral tilt as a function of the nonminimal coupling parameter $\xi_{\chi}$. The other parameters are set to $\xi_{h}=65000$ and $\lambda=1$. Note, however, that changing the ratio $\xi_{h} / \sqrt{\lambda}$ in the observationally allowed range affects the result only by a negligible amount. The solid curves correspond to the numerical results, the blue (lower) one is obtained for $\varrho_{r h}=$ $\varrho_{r h}^{\min }$ (long reheating) and the red (upper) one for $\varrho_{r h}=\varrho_{r h}^{\max }$ (instantaneous reheating). The blue (lower) and red (upper) dashed curves are obtained from the analytical approximation (116) for $\bar{N}_{\min }^{*}$ and $\bar{N}_{\max }^{*}$. The straight dashed line represents the asymptotic solution (133), which is a good approximation if $\frac{1}{4 N^{*}}<\xi_{\chi} \ll 1$. The horizontal line and the shaded regions correspond to the observational mean value and the $1 \sigma$ and $3 \sigma$ confidence intervals, cf. Eq. (105).

We have found that the parameters need to satisfy $\xi_{\chi} \ll 1$ and $\xi_{h} \gg 1$. With this knowledge, we again carry out the above four steps and derive approximate analytical results. From Eq. (86), we obtain

$$
\theta_{\text {end }}=2 \times 3^{1 / 4} \sqrt{\xi_{\chi}}\left(1+\mathcal{O}\left[\xi_{\chi}, \frac{1}{\xi_{h}}\right]\right) .
$$

In order to approximately solve Eq. (85) for $\theta^{*}$, we can neglect the second term on the right-hand side. The inversion then gives

$$
\theta^{*} \simeq \arccos \left(\cos \left(\theta_{\text {end }}\right) e^{-4 \xi_{X} N^{*}}\right) .
$$

Here, the sign for approximate equality " $\simeq$ " refers to the approximation made when inverting Eq. (85). This approximation will constitute the main source of error in the approximate expressions for the spectral quantities. One can get a more accurate approximation by reinserting the first approximation into the right-hand side of Eq. (85) in order to compute the second-order approximation of an iterative solution. However, as the expressions get considerably more complicated, we stick to the first-order approximation, which already comes very close to the numerical results. Inserting $\theta_{\text {end }}$ from Eq. (112) into Eq. (113), one obtains

$$
\theta^{*} \simeq \arccos \left(e^{-4 \xi_{\chi} N^{*}}\right)\left(1+\mathcal{O}\left[\xi_{\chi}, \frac{1}{\xi_{h}}\right]\right) .
$$

We can now evaluate the spectral parameters at the approximate value for $\theta^{*}$. Inserting Eq. (114) into Eqs. (97)-(99) and (102) and recalling Eq. (103) we obtain ${ }^{18}$

$$
\begin{gathered}
P_{\zeta}\left(k_{0}\right) \simeq \frac{\lambda \sinh ^{2}\left(4 \xi_{\chi} N^{*}\right)}{1152 \pi^{2} \xi_{\chi}^{2} \xi_{h}^{2}}\left(1+\mathcal{O}\left[\xi_{\chi}, \frac{1}{\xi_{h}}, \frac{1}{N^{*}}\right]\right) \\
n_{s}\left(k_{0}\right)-1 \simeq-8 \xi_{\chi} \operatorname{coth}\left(4 \xi_{\chi} N^{*}\right)\left(1+\mathcal{O}\left[\xi_{\chi}, \frac{1}{\xi_{h}}, \frac{1}{N^{*}}\right]\right), \\
\alpha_{\zeta}\left(k_{0}\right) \simeq-32 \xi_{\chi}^{2} \sinh ^{-2}\left(4 \xi_{\chi} N^{*}\right)\left(1+\mathcal{O}\left[\xi_{\chi}, \frac{1}{\xi_{h}}, \frac{1}{N^{*}}\right]\right),
\end{gathered}
$$

$$
\begin{aligned}
r\left(k_{0}\right) & =-8 n_{g}\left(k_{0}\right) \\
& \simeq 192 \xi_{\chi}^{2} \sinh ^{-2}\left(4 \xi_{\chi} N^{*}\right)\left(1+\mathcal{O}\left[\xi_{\chi}, \frac{1}{\xi_{h}}, \frac{1}{N^{*}}\right]\right) .
\end{aligned}
$$

One can see that in this approximation, $\alpha_{\zeta}\left(k_{0}\right), r\left(k_{0}\right)$ and $n_{g}\left(k_{0}\right)$ are related as

$$
\alpha_{\zeta}\left(k_{0}\right) \simeq-\frac{1}{6} r\left(k_{0}\right)=\frac{4}{3} n_{g}\left(k_{0}\right),
$$

which can be understood as an approximate consistency condition for the Higgs-dilaton model.

In order to find $N^{*}$ in terms of the parameters of the theory, we insert Eq. (114) into Eq. (107), from which we derive the approximate results ${ }^{19}$

$$
N_{\text {min }}^{*} \simeq\left(64.3-\frac{1}{12} \ln \lambda-\frac{2}{3} \ln \frac{\xi_{h}}{\sqrt{\lambda}}\right)\left(1+\mathcal{O}\left[\xi_{\chi}, \frac{1}{\xi_{h}}, \frac{1}{N^{*}}\right]\right),
$$

$$
N_{\max }^{*} \simeq\left(64.5-\frac{1}{2} \ln \frac{\xi_{h}}{\sqrt{\lambda}}\right)\left(1+\mathcal{O}\left[\xi_{\chi}, \frac{1}{\xi_{h}}, \frac{1}{N^{*}}\right]\right),
$$

where the subscripts "min" and "max" stand for the cases $\varrho_{r h}=\varrho_{r h}^{\min }$ and $\varrho_{r h}=\varrho_{r h}^{\max }$, respectively. These approximate results, together with the numerical results for $N_{\text {min }}^{*}$ and $N_{\max }^{*}$, are plotted in Fig. 6.

Equations (115)-(118), together with Eqs. (120) and (121), constitute our approximate analytical results for the spectral parameters in terms of the parameters $\xi_{\chi}, \xi_{h}$, and $\lambda$, in the two limiting cases of instantaneous and late reheating. One can see that the results depend on $\xi_{h}$ and $\lambda$ mainly through the combination $\xi_{h} / \sqrt{\lambda}$. Independent variation of $\lambda$ affects the spectral parameters only through $N_{\min }^{*}$. Further, we remark that for $0.1<\lambda<1$ and $\xi_{h} / \sqrt{\lambda}$

\footnotetext{
${ }^{18}$ Note that these approximate results can equivalently be derived from the approximate action given by Eqs. (50) and (51) with $\alpha=0$.

${ }^{19}$ The numerical factor is given to the first decimal.
} 

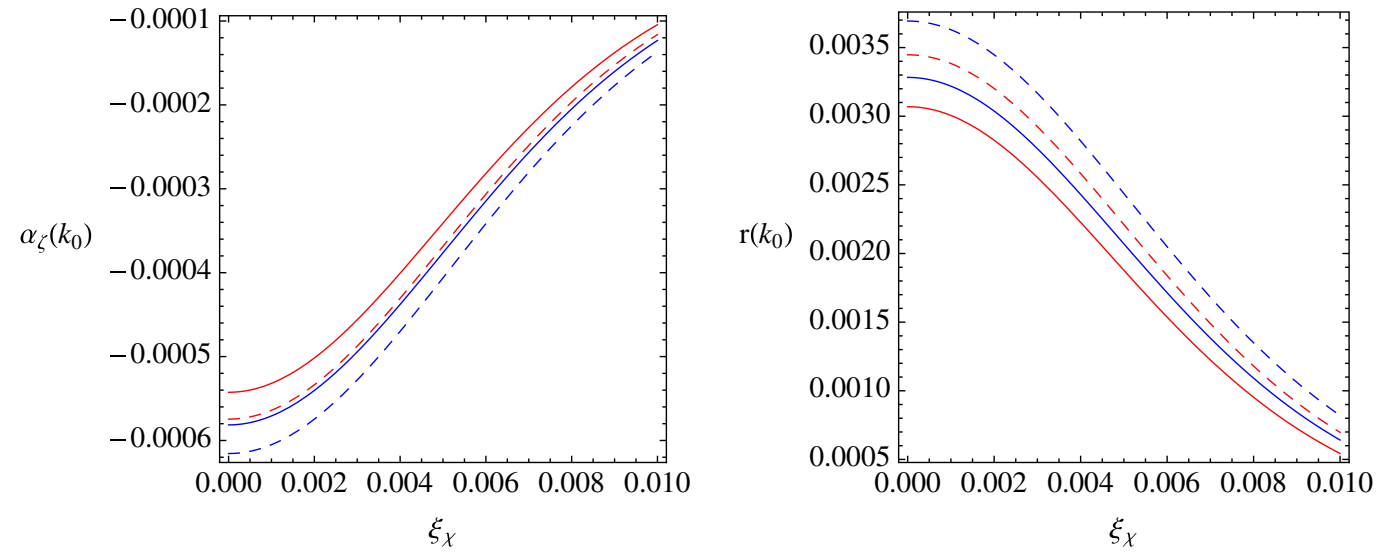

FIG. 5 (color online). The running of the scalar spectral tilt (left) and the tensor-to-scalar ratio (right) as a function of the coupling $\xi_{\chi}$. The other parameters are set to $\xi_{h}=65000$ and $\lambda=1$. Note, however, that changing the ratio $\xi_{h} / \sqrt{\lambda}$ in the observationally allowed range affects the result only by a negligible amount. Solid curves show the numerical results, while dashed curves are obtained from the approximate expressions(118) and (117). In the plot on the left, the red (upper) solid and dashed curves show the case of instant reheating, and the blue (lower) curves show the case of long reheating. In the plot on the right, the red (lower) solid and dashed curves show the case of instant reheating, and the blue (upper) curves show the case of long reheating.

in the observationally allowed range of Eq. (110) or Eq. (111), both $N_{\min }^{*}$ and $N_{\max }^{*}$ vary only very little with the parameters (cf. Fig. 6). For the precision required here, it is enough to know $N^{*}$ at the precision of a whole number. Therefore, inserting parameters of the allowed order of magnitude, we can set the approximate values to

$$
\begin{aligned}
& \bar{N}_{\text {min }}^{*}=57, \\
& \bar{N}_{\max }^{*}=59 .
\end{aligned}
$$

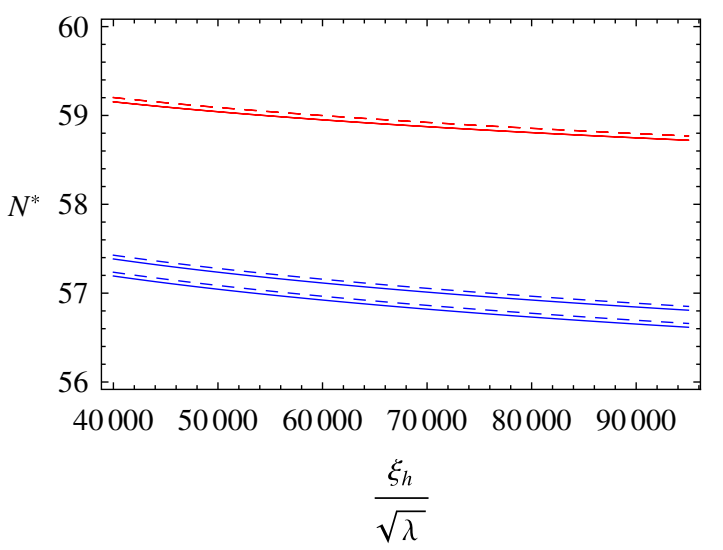

FIG. 6 (color online). The number of e-folds $N^{*}$ between horizon crossing of the scale $k_{0}$ and the end of inflation as a function of $\xi_{h} / \sqrt{\lambda}$, for $\xi_{\chi}=0.001$. Note that changing $\xi_{\chi}$ in the observationally allowed range affects the result by a negligible amount. The solid curves correspond to the numerical results, the blue (lower two) ones correspond to $N_{\min }^{*}$ (long reheating), and the red (top) one to $N_{\text {max }}^{*}$ (instant reheating). Among the blue (lower two) curves, the upper one is obtained for $\lambda=1$ and the lower one for $\lambda=0.1$, showing the slight dependence of $N_{\min }^{*}$ on $\lambda$. The dependence of $N_{\max }^{*}$ on $\lambda$ is negligible. The dashed curves are obtained from the analytical approximations (120) and (121).
In the two limiting cases, the spectral parameters can be evaluated at these values for $N^{*}$. Neglecting the small variation of $N^{*}$ with the parameters of the theory, we observe that the amplitude $P_{\zeta}$ of the scalar spectrum depends on the combination $\xi_{h} / \sqrt{\lambda}$ and on $\xi_{\chi}$. The expression (115) allows to understand the shape of the allowed parameter region in Fig. 3. The other spectral parameters, unlike the amplitude, are practically independent of $\xi_{h}$ and $\lambda$ and depend on the single parameter $\xi_{\chi}$. Hence, we can plot $n_{s}, \alpha_{\zeta}$, and $r$ as functions of $\xi_{\chi}$ (cf. Figs. 4 and 5). Compared to the numerical results, the approximate formula for the spectral tilt has an accuracy of the order of $10^{-3}$, while the accuracy of the approximate results for $\alpha_{\zeta}$ and $r$ is of the order of $5 \cdot 10^{-4}$. Given the uncertainties in the observational values, these accuracies are largely sufficient.

As long as the quantity $4 \xi_{\chi} N^{*}$ is smaller than 1 (i.e. $\left.\xi_{\chi} \lesssim 0.004\right)$, the series expansions of the hyperbolic functions in Eqs. (115)-(118) converge rapidly, and the expressions can be further approximated by

$$
\begin{gathered}
P_{\zeta}\left(k_{0}\right) \simeq \frac{\lambda N^{* 2}}{72 \pi^{2} \xi_{h}^{2}}\left(1+\frac{1}{3}\left(4 \xi_{\chi} N^{*}\right)^{2}+\ldots\right), \\
n_{s}\left(k_{0}\right)-1 \simeq-\frac{2}{N^{*}}\left(1+\frac{1}{3}\left(4 \xi_{\chi} N^{*}\right)^{2}+\ldots\right), \\
\alpha_{\zeta}\left(k_{0}\right) \simeq-\frac{2}{N^{* 2}}\left(1-\frac{1}{3}\left(4 \xi_{\chi} N^{*}\right)^{2}+\ldots\right), \\
r\left(k_{0}\right) \simeq \frac{12}{N^{* 2}}\left(1-\frac{1}{3}\left(4 \xi_{\chi} N^{*}\right)^{2}+\ldots\right) .
\end{gathered}
$$


From these expressions, we can see that in the limit $\xi_{\chi} \rightarrow 0$, the predictions of the Higgs-dilaton inflation model reduce to those found for the Higgs-Inflation model [31]. Hence, one can think of $\xi_{\chi}$ as the deviation of our predictions from those of the Higgs-Inflation model. In the Higgs-dilaton scenario, the results for $n_{s}, \alpha_{\zeta}$, and $r$ in the limit $\xi_{\chi} \rightarrow 0$ constitute a prediction of bounds on these quantities. We find (cf. Figs. 4 and 5)

$$
\begin{gathered}
n_{s}\left(k_{0}\right)<0.97 \simeq 1-\frac{2}{N^{*}}, \\
\alpha_{\zeta}\left(k_{0}\right)>-0.0006 \simeq-\frac{2}{N^{* 2}}, \\
r\left(k_{0}\right)<0.0033 \simeq \frac{12}{N^{* 2}} .
\end{gathered}
$$

These bounds are nontrivial predictions of our model. Further, given that $n_{s}, \alpha_{\zeta}$, and $r$ are functions of $\xi_{\chi}$ only, the bound $\xi_{\chi} \lesssim 0.008$ deduced from the observational lower bound on $n_{s}$ translates to (cf. Fig. 5)

$$
\begin{gathered}
\alpha_{\zeta}\left(k_{0}\right) \lesssim-0.00015, \\
r\left(k_{0}\right) \gtrsim 0.0009 .
\end{gathered}
$$

The upper bound on $n_{s}$ is well in accord with the observational constraints. Results of the Planck mission are expected to reduce the errors by a factor of a few and will, hence, provide an important test of the Higgs-dilaton model [74]. While the present observational limits on $\alpha_{\zeta}$ and $r$ are too weak to compete with the bounds derived above [64], the results of Planck might also improve this situation.

We will see in Sec. IVA that, if the scalar fields constitute a dark-energy component at late times, its equation of state parameter $w_{\mathrm{DE}}^{0}$ is also a function of the parameter $\xi_{\chi}$ only. As a consequence, the observational lower bound on $n_{s}$ will induce a bound on $w_{\mathrm{DE}}^{0}$. Thereby, the Higgsdilaton model provides a nontrivial connection between observables related to the early and the late Universe.

Besides the parameter region in which both $\xi_{X}$ and $4 \xi_{\chi} N^{*}$ are small, the observational bounds do not completely exclude the region where $\xi_{\chi}$ is small, but $4 \xi_{\chi} N^{*}$ is somewhat bigger than unity. In this region, as can be deduced from the approximate results (116)-(118), the predicted values for $\alpha_{\zeta}$ and $r$ go to zero exponentially with growing $4 \xi_{\chi} N^{*}$, while the spectral tilt becomes asymptotically linear in $\xi_{\chi}$ (cf. Fig. 4), i.e.

$$
n_{s}\left(k_{0}\right)-1 \simeq-8 \xi_{\chi}, \quad \text { for } \quad \frac{1}{4 N^{*}}<\xi_{\chi} \ll 1 .
$$

This fact will allow us to speculate about a somewhat deeper connection between $n_{s}$ and $w_{\mathrm{DE}}$ in Sec. IVA.
From the approximate results (116)-(118), one can see that $\alpha_{\zeta}$ and $r$ are suppressed with respect to $n_{s}-1$. In the region where $4 \xi_{\chi} N^{*}<1$, the suppression factor is $1 / N^{*}$, while in the region where $4 \xi_{\chi} N^{*}>1$, the suppression factor is smaller than $\xi_{\chi}$. This fact justifies the use of the observational bounds (104) and (105), that are based on the assumption of a power-law spectrum for scalar perturbations and the absence of tensor modes.

The above results provide limits on the reheating temperature $T_{r h}$, defined as the initial temperature of the homogeneous radiation-dominated Universe. $T_{r h}$ is related to $\varrho_{r h}$ through

$$
\varrho_{r h}=\frac{\pi^{2}}{30} g_{\text {eff }}\left(T_{r h}\right) T_{r h}^{4},
$$

where $g_{\text {eff }}\left(T_{r h}\right)$ is the effective number of relativistic degrees of freedom present in the thermal bath at the temperature $T_{r h}$. Counting all degrees of freedom of the standard model plus the dilaton, one has $g_{\text {eff }}\left(T_{r h}\right)=$ 107.75. To lowest nontrivial order in $\xi_{\chi}$ and $1 / \xi_{h}$, the limits on $\varrho_{r h}$, i.e. $V\left(\theta_{r h}^{\min }\right)<\varrho_{r h}<V\left(\theta_{\text {end }}\right)$ (cf. Eqs. (108) and (109)) are found to be

$$
\begin{gathered}
V\left(\theta_{r h}^{\min }\right) \simeq \frac{\lambda}{144 \xi_{h}^{4}} M_{P}^{4}, \\
V\left(\theta_{\mathrm{end}}\right) \simeq \frac{\lambda}{\xi_{h}^{2}} X M_{P}^{4},
\end{gathered}
$$

where $X=7-4 \sqrt{3} \simeq 0.7$. The bounds on $\varrho_{r h}$, together with the obtained bounds on $\xi_{h} / \sqrt{\lambda}$ (110) and (111), translate into the following bounds on the reheating temperature $T_{r h}$ :

$$
3.8 \sqrt{\frac{65000}{\xi_{h}}} \cdot 10^{12} \mathrm{GeV} \lesssim T_{r h} \lesssim 2.5 \times 10^{15} \mathrm{GeV} .
$$

Finally, let us note that the findings of this section allow us to constrain the region of initial conditions for the scalar fields, which lead to successful inflation. Based on the assumption that the last $N^{*}$ e-folds of inflation take place in the scale-invariant region, we have found the field value $\theta^{*}$ close to which the observable scales exit the Hubble horizon during inflation. The initial conditions for inflation have to be such that $\theta_{\text {in }} \geq \theta^{*}$. In terms of the original variables, this condition reads

$$
\frac{h_{\text {in }}}{\chi_{\text {in }}} \geq \sqrt{\frac{1+6 \xi_{\chi}}{1+6 \xi_{h}}} \tan \theta^{*} .
$$

$\theta^{*}$ was found to be $\theta^{*} \simeq \arccos \left(e^{-4 \xi_{X} N^{*}}\right)$ (114). For typical parameter values $\xi_{\chi}=0.005, \xi_{h}=65000$, and $N^{*}=58$, one obtains $\theta^{*} \simeq 1.25$ and $\frac{h_{\text {in }}}{\chi_{\text {in }}} 0.3 \gtrsim 0.005$. Considerations 


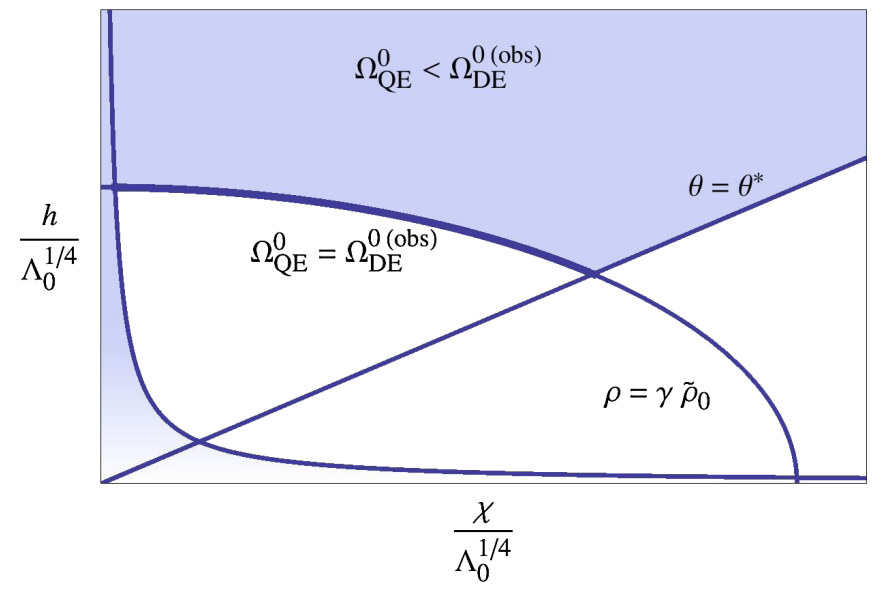

FIG. 7 (color online). This plot shows the different regions of initial conditions giving rise to qualitatively different evolutions. For a successful description of inflation, initial conditions have to lie above the line $\theta=\theta^{*} \simeq \arccos \left(e^{-4 \xi_{X} N^{*}}\right)$. For $\Lambda_{0}>0$, the scalar fields contribute to dark energy in the late stage. Initial conditions have to lie above the arc of an ellipse given by $\rho \simeq$ $\gamma \tilde{\rho}_{0} \simeq-\frac{M_{P}}{4} \ln \left(\gamma^{4} \frac{\Lambda_{\text {eff }}}{\Lambda_{0}}\right)$ for this contribution not to exceed the observed value of $\Omega_{\mathrm{DE}}^{0}$. Hence, the blue (shaded) region corresponds to initial conditions giving rise to successful inflation and a contribution to dark energy not exceeding $\Omega_{\mathrm{DE}}^{0 \text { (obs) }}=0.74$. The bold segment of the ellipse corresponds to initial conditions for which the scalar fields yield the total observed dark energy. The hyperbola is given by $v_{2}=1$. Initial conditions below the hyperbola lie in the non-scale-invariant region, where $\Lambda_{0}$ is important. Trajectories starting here tend to move away from the origin before entering the scale-invariant region and following a scale-invariant trajectory. Therefore, such initial conditions can also be acceptable as long as the corresponding trajectories enter the scale-invariant region at or above the line given by $\rho$. Note that, while we only describe the quadrant $\chi / \Lambda_{0}^{1 / 4}$, $h / \Lambda_{0}^{1 / 4}>0$, the reasoning would be completely analog in the other quadrants.

related to dark energy (Sec. IV B) will yield an additional constraint on the initial conditions. The region of acceptable initial conditions satisfying both constraints is shown in Fig. 7.

\section{IMPLICATIONS FOR THE LATE UNIVERSE}

We have shown in the previous section that a number of parameters of the theory can be constrained by two independent inflationary observables: the amplitude and the tilt of the primordial spectrum of scalar perturbations $\mathcal{P}_{\zeta}$, cf. Fig. 3. The theory is, therefore, completely specified at the inflationary stage, and any subsequent period should be consistent with that choice of parameters. In this section, we focus on the late dark-energy dominated stage previously described in Sec. II C, during which the dilaton field is rolling down along one of the potential valleys. In Sec. IVA, we show how this results in the dilaton playing the role of a quintessence field. We then derive consistency conditions among the inflationary observables and those associated to the dark-energy dominated stage, which could allow us to either confirm or exclude the model in the coming years. In Sec. IV B, we derive a constraint that has to be imposed on the initial conditions of the scalar fields in order to have a successful description of dark energy. This will prove a posteriori that the whole period of observable inflation must take place in the scaleinvariant region.

\section{A. The dilaton as quintessence field}

After the phase of reheating, the system enters the radiation-dominated stage, at the beginning of which the total energy density is given by $\varrho_{r h}$ (cf. Eq. (134)). At that moment, the scalar fields have almost settled down in one of the potential valleys, i.e. $h(t)^{2} \simeq \frac{\alpha}{\lambda} \chi(t)^{2}$ or, in terms of the variables $(\tilde{\rho}, \tilde{\theta}), \tanh ^{2}\left(a \tilde{\theta}(t) / M_{P}\right) \simeq$ $\frac{1-\varsigma}{1+\frac{\alpha 1+6 \xi_{h}}{\lambda 1+6 \xi_{x}}}=1-\varsigma+\mathcal{O}(\alpha)$.

We will work with the assumption that the equality is exact and that the fields evolve exactly along the valley (cf. [1]). ${ }^{20}$ In this case, at the level of homogeneous fields, we are left with a single degree of freedom $\tilde{\rho}(t)$. As discussed in Sec. II, also at the level of perturbations, the field $\tilde{\rho}$ (or equivalently $\rho$ ) is almost decoupled from the SM fields. Hence, we will from now on treat $\tilde{\rho}$ as a field minimally coupled to gravity and not interacting with matter and radiation. Given that $\mu \ll 1$ and $\alpha \ll 1$, its dynamics is described by the E-frame Lagrangian (25) with $\tilde{K}$ and $\tilde{U}=\tilde{V}+\tilde{V}_{\Lambda_{0}}$ given by Eqs. (50)-(52), where one inserts the constraint $\tanh ^{2}(a \tilde{\theta}(t) / M) \simeq 1-$ s, i.e.

$$
\frac{\mathcal{L}}{\sqrt{-\tilde{g}}} \simeq \frac{M_{P}^{2}}{2} \tilde{R}-\frac{1}{2}(\partial \tilde{\rho})^{2}-\tilde{V}_{\mathrm{QE}}(\tilde{\rho})
$$

with

$$
\tilde{V}_{\mathrm{QE}}(\tilde{\rho})=\frac{\Lambda_{0}}{\gamma^{4}} e^{-4 \gamma \tilde{\rho} / M_{P}}
$$

As already pointed out in [1], it is remarkable that the exponential potential, which was proposed for $\mathrm{QE}$ a long time ago $[34,35,75]$, appears automatically in the present model. It, hence, turns out that the dilaton field $\tilde{\rho}$ can play the role of QE.

Let us now discuss in more detail the influence of the field $\tilde{\rho}$ on standard homogeneous cosmology. The equation of motion for the homogeneous field $\tilde{\rho}=\tilde{\rho}(t)$ in spatially flat FLRW space-time is given by

\footnotetext{
${ }^{20}$ The validity of this approximation can be checked numerically. Let us further note that the trajectory going exactly along the valley is an asymptotic, but not an exact solution of the equations of motion.
} 


$$
\ddot{\tilde{\rho}}+3 H \dot{\tilde{\rho}}+\frac{d V_{\mathrm{QE}}}{d \tilde{\rho}}=0 .
$$

Defining energy density $\varrho_{\mathrm{QE}}$, pressure $p_{\mathrm{QE}}$, and equation of state parameter $w_{\mathrm{QE}}$ of the scalar-field $\tilde{\rho}$ as

$$
\begin{gathered}
\varrho_{\mathrm{QE}} \equiv \frac{1}{2} \dot{\tilde{\rho}}^{2}+V_{\mathrm{QE}}, \\
p_{\mathrm{QE}} \equiv \frac{1}{2} \dot{\tilde{\rho}}^{2}-V_{\mathrm{QE}}, \\
w_{\mathrm{QE}} \equiv \frac{p_{\mathrm{QE}}}{\varrho_{\mathrm{QE}}}
\end{gathered}
$$

its equation of motion (141) can equivalently be written as

$$
\dot{\varrho}_{\mathrm{QE}}=-3 H \varrho_{\mathrm{QE}}\left(1+w_{\mathrm{QE}}\right) .
$$

On the other hand, in the presence of a barotropic fluid of energy density $\varrho_{b}$, for instance relativistic or nonrelativistic matter, the Hubble parameter is given by the first Friedmann equation as

$$
H^{2}=\frac{1}{3 M_{P}^{2}}\left(\varrho_{b}+\varrho_{\mathrm{QE}}\right),
$$

which, in terms of the relative abundances $\Omega=$ $\varrho / 3 M_{P}^{2} H^{2}$, can be written as the cosmic sum rule $\Omega_{b}+$ $\Omega_{\mathrm{QE}}=1$. The cosmological model described by Eqs. (141) and (146) with a scalar-field evolving in an exponential potential has been widely studied in the literature (for a recent review see [76]). We want to recap the main results established in the literature and show how they apply to our model.

For the qualitative analysis of the system we rewrite Eqs. (141) and (146) in terms of the observable quantities $\Omega_{\mathrm{QE}}$ and $\delta_{\mathrm{QE}} \equiv 1+w_{\mathrm{QE}}$ as (cf. e.g. [77])

$$
\begin{gathered}
\delta_{\mathrm{QE}}^{\prime}=-3 \delta_{\mathrm{QE}}\left(2-\delta_{\mathrm{QE}}\right)+4 \gamma\left(2-\delta_{\mathrm{QE}}\right) \sqrt{3 \delta_{\mathrm{QE}} \Omega_{\mathrm{QE}}}, \\
\Omega_{\mathrm{QE}}^{\prime}=3\left(\delta_{b}-\delta_{\mathrm{QE}}\right) \Omega_{\mathrm{QE}}\left(1-\Omega_{\mathrm{QE}}\right),
\end{gathered}
$$

where prime, as before, denotes the derivative with respect to the number of e-folds $N=\ln a$. Further, $\delta_{b} \equiv 1+w_{b}$, where $w_{b}$ is the equation of state parameter of the barotropic fluid. For radiation, one has $\delta_{b}=4 / 3$, while for nonrelativistic matter, $\delta_{b}=1$. An additional dark-energy component with constant equation of state would have $\delta_{b}<2 / 3$. In the scale-invariant model analyzed here, a component of this type is present as soon as the action (11) contains a term $\beta \chi^{4}$ with $\beta>0$ (cf. also Sec. II A). For the reasons mentioned in Sec. II A 3, we will mainly focus on the case $\beta=0$, in which only $\tilde{\rho}$ will be responsible for dark energy. ${ }^{21}$ It has been shown in $[34,36,78]$ that as long as $0 \leq \delta_{b} \leq 2$ and depending on the value of the

\footnotetext{
${ }^{21}$ Let us mention that, even in the case $\beta<0$, appropriate initial conditions lead to a dark-energy dominated phase.
}

parameter $\gamma$, the system approaches one of two qualitatively very different attractor solutions.

For $4 \gamma>\sqrt{3 \delta_{b}}$, the variables evolve towards the stable fixed point $\Omega_{\mathrm{QE}}=3 \delta_{b} / 16 \gamma^{2}$ and $\delta_{\mathrm{QE}}=\delta_{b}$. This means that the scalar field inherits the equation of state parameter of the barotropic fluid. Hence, the energy density of the scalar-field scales like the energy density of the fluid. Unless the scalar field gives the dominating contribution to the energy density from the very beginning, it will never become dominating. Therefore, these so-called "scaling solutions" cannot be responsible for the late-time acceleration of the Universe. In this case, the accelerated expansion must be due to another mechanism, e.g. a barotropic dark-energy component with $\delta_{b}<2 / 3$. In other words, a scaling field can at best provide a small contribution to dark energy.

For $4 \gamma<\sqrt{3 \delta_{b}}$, the situation is very different. The stable fixed point is given by $\Omega_{\mathrm{QE}}=1$ and $\delta_{\mathrm{QE}}=16 \gamma^{2} / 3$. Hence, in this case, the asymptotic solution describes a scalar-fielddominated universe, which is accelerating if $4 \gamma<\sqrt{2}$, i.e. $\xi_{\chi} \lesssim \frac{1}{2}$. This means that the scalar field with exponential potential and $4 \gamma<\sqrt{3 \delta_{b}}$ can describe the late-time acceleration of the Universe, provided that the system has not quite reached the fixed point by today. ${ }^{22}$

In the previous section, we have found that our model can successfully describe inflation if $\xi_{\chi} \leqslant 8 \times 10^{-3}$. This yields the bound $4 \gamma \simeq 4 \sqrt{\xi_{\chi}} \lessgtr 0.36$. Hence, for this parameter choice, the system evolves toward the second type of fixed point, corresponding to a scalar-field-dominated universe in accelerated expansion. This allows us to draw a nontrivial conclusion. Namely, if the parameters of the model are fixed by the requirements of inflation, and for $\beta=0$, the late-time behavior of the system necessarily corresponds to an accelerating universe, dominated by $\tilde{\rho}$.

Current observations [64] show that the present abundance of dark energy is $\Omega_{\mathrm{DE}}^{0} \simeq 0.74$. For $\beta=0$, dark energy is entirely due to $\tilde{\rho}$, and we can identify $\Omega_{\mathrm{QE}}^{0}=$ $\Omega_{\mathrm{DE}}^{0}$ and $w_{\mathrm{QE}}^{0}=w_{\mathrm{DE}}^{0}$. The observed value shows that dark energy is not clearly dominating the present Universe, which means that the system must not have reached its fixed point yet.

We now want to qualitatively discuss the scenario in which the field $\tilde{\rho}$ is irrelevant during the radiation- and matter-dominated stages, but has become important recently and is now responsible for the present acceleration of the Universe. During the radiation- and matterdominated stages, one must have $\Omega_{\mathrm{QE}} \ll 1$. As long as this is the case, the second term on the right-hand side of Eq. (147) is small compared to the first one. Hence, $\delta_{\mathrm{QE}}$ is driven toward a very small value $\delta_{\mathrm{QE}} \ll 1$ and $w_{\mathrm{QE}} \simeq-1$. This shows that, even if initially $\varrho_{\mathrm{QE}}$ were

\footnotetext{
${ }^{22}$ As has been shown in [79], this statement holds even if $4 \gamma>\sqrt{2}$.
} 
dominated by kinetic energy, the kinetic part would soon die away and $\Omega_{\mathrm{QE}}$ become potential dominated. ${ }^{23}$ As a consequence, the value of $\tilde{\rho}$ is almost constant during the radiation- and matter-dominated epochs and remains practically equal to its value at the end of reheating. However, since $\varrho_{\mathrm{QE}}$ decreases more slowly than the energy densities of radiation and matter, $\Omega_{\mathrm{QE}}$ becomes relevant at some point. At this point, $\tilde{\rho}$ starts rolling down the potential, and $\delta_{\mathrm{QE}}$ starts growing toward its attractor value. The initial value of $\Omega_{\mathrm{QE}}$ has to be small enough such that $\Omega_{\mathrm{QE}}$ remains negligible throughout radiation domination and only becomes important in the late matter-dominated stage. The described scenario in which the quintessence field remains constant for a long time and then starts rolling down the potential goes under the name of "thawing quintessence" [37]. Two recent studies treating the case of an exponential potential can be found in $[77,80]$.

In the approximation where $\delta_{\mathrm{QE}}=1+w_{\mathrm{QE}} \ll 1$, the system of equations (147) and (148) can be integrated, and one finds the interesting relation [77]

$$
1+w_{\mathrm{QE}} \simeq \frac{16 \gamma^{2}}{3} F\left(\Omega_{\mathrm{QE}}\right)
$$

where

$$
F\left(\Omega_{\mathrm{QE}}\right)=\left[\frac{1}{\sqrt{\Omega_{\mathrm{QE}}}}-\frac{1}{2}\left(\frac{1}{\Omega_{\mathrm{QE}}}-1\right) \ln \frac{1+\sqrt{\Omega_{\mathrm{QE}}}}{1-\sqrt{\Omega_{\mathrm{QE}}}}\right]^{2} .
$$

The function $F\left(\Omega_{\mathrm{QE}}\right)$ is monotonically increasing from 0 to 1 (cf. Fig. 8). For the observed value $\Omega_{\mathrm{QE}}^{0}=\Omega_{\mathrm{DE}}^{0} \simeq$ 0.74 , one gets $F\left(\Omega_{\mathrm{QE}}^{0}=0.74\right) \simeq 0.5$. Inserting this into Eq. (149) and identifying $w_{\mathrm{QE}}^{0}=w_{\mathrm{DE}}^{0}$, we obtain the following result for the present equation of state parameter of dark energy (provided by quintessence field $\tilde{\rho}$ )

$$
1+w_{\mathrm{DE}}^{0} \simeq \frac{8}{3} \frac{\xi_{\chi}}{1+6 \xi_{\chi}} .
$$

We can now plug into this relation the upper bound $\xi_{\chi} \lesssim$ 0.008 (cf. Eq. (111)), derived from the observational bound on $n_{s}$, as well as the theoretical lower bound $\xi_{\chi}>0$, such that (cf. Fig. 9)

$$
0 \leq 1+w_{\mathrm{DE}}^{0} \leq 0.02
$$

Thus, we have found that the parameter bound from inflation implies a strong bound on the equation of state

\footnotetext{
${ }^{23}$ In principle, one could imagine a scenario in which, after reheating, $\Omega_{\mathrm{QE}}$ is non-negligible, as long as $\delta_{\mathrm{QE}} \simeq 1$. Since the kinetic part of $\varrho_{\mathrm{QE}}$ decreases as $a^{-6}$, it would soon fall below $\varrho_{\text {radiation }}$, and radiation would start dominating, provided that the potential energy of $\tilde{\rho}$ is small enough. However, we do not expect this to happen in our model, because the field $\tilde{\rho}$ is almost constant during reheating.
}

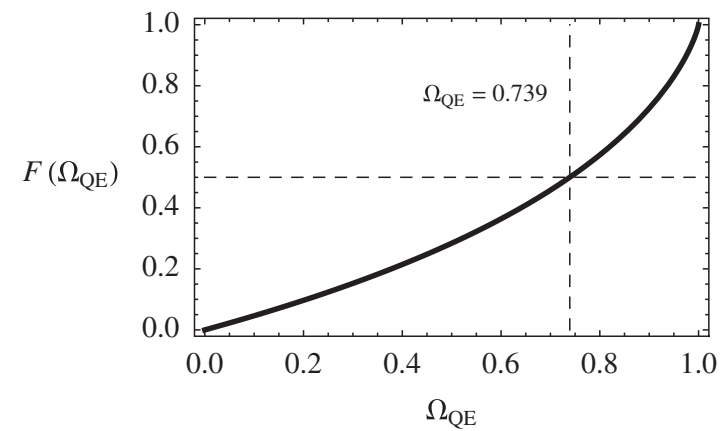

FIG. 8. The function $F\left(\Omega_{\mathrm{QE}}\right)$. Note that it becomes exactly $1 / 2$ for $\Omega_{\mathrm{QE}}=0.739$, which is very close to the observed abundance of dark energy $\Omega_{\mathrm{DE}}^{0}=0.725 \pm 0.048$ (WMAP7 $+\mathrm{BAO}+H_{0}$ at $99 \%$ confidence level [64]). At this value, the functional relation between the spectral tilt $n_{s}$ of CMB anisotropies and the equation of state parameter $w_{\mathrm{QE}}^{0}$ is particularly simple.

parameter of dark energy. This is a rather nontrivial result. The current observational constraint $-0.52<1+w_{\mathrm{DE}}^{0}<$ 0.32 (WMAP7 $+\mathrm{BAO}+H_{0}$ at $99 \%$ confidence level [64]) is much too weak to compete with this theoretical prediction. From this point of view, the energy density $\varrho_{\mathrm{QE}}$ is practically indistinguishable from a cosmological constant. Nevertheless, the observational bound is expected to improve considerably in the near future. While the Dark Energy Survey collaboration aims at measurement of $w_{\mathrm{DE}}^{0}$ with an accuracy of $\sim 5 \%$ [81], the expected accuracy from the Euclid consortium is 2\% [82]. These measurements, together with the projected improvement on the determination of $n_{s}$ from the Planck mission [74], should provide an important consistency check of the Higgs-dilaton model in the near future.

In fact, the theoretical predictions of our model (with $\beta=0$ ) can be further refined. Namely, since both the scalar spectral index $n_{s}$ and the equation of state parameter $w_{\mathrm{DE}}^{0}$ depend mainly on $\xi_{\chi}$, it is possible to establish a functional relation between these two very different observables. Combining Eq. (151) with the approximate relation (116) allows us to express the scalar tilt $n_{s}$ as a function of $\delta_{\mathrm{DE}}^{0}$ and the number of e-folds $N^{*}$ as

$$
n_{s}-1 \simeq-\frac{12 \delta_{\mathrm{DE}}^{0}}{4-9 \delta_{\mathrm{DE}}^{0}} \operatorname{coth}\left(\frac{6 N^{*} \delta_{\mathrm{DE}}^{0}}{4-9 \delta_{\mathrm{DE}}^{0}}\right)
$$

We plot this relation and the corresponding numerical result in Fig. 9. The plot is equivalent to the plot of Fig. 4, except that the independent variable is changed from $\xi_{\chi}$ to $\delta_{\mathrm{DE}}=1+w_{\mathrm{DE}}^{0}$ with the help of Eq. (151). As before, we see that the result is rather insensitive to variations of $N^{*}$ in the range $\bar{N}_{\min }^{*}<N^{*}<\bar{N}_{\max }^{*}$, cf. Eqs. (122) and (123). One can also derive a relation involving the respective second-order quantities 


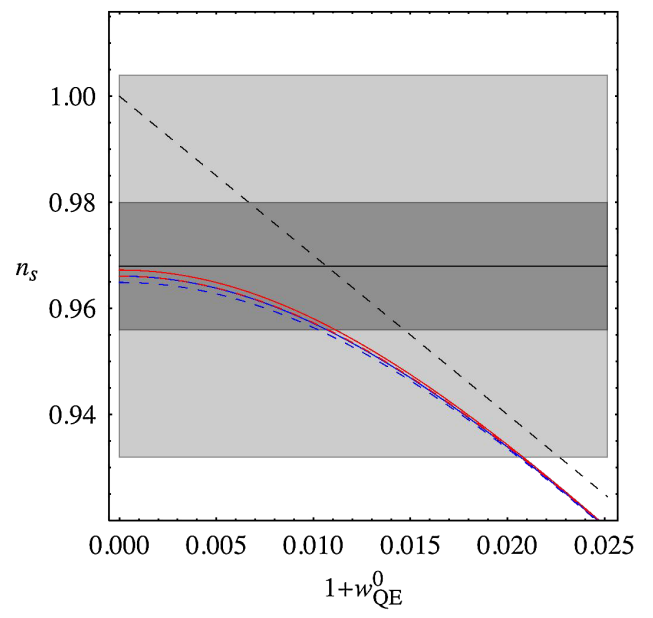

FIG. 9 (color online). This plot shows the approximate functional relationship between $n_{s}$ and $w_{\mathrm{DE}}^{0}$. The plain curves are numerical results. The red (upper) one is obtained for $\varrho_{r h}=$ $\varrho_{r h}^{\max }$ (instant reheating), while the blue (lower) one represents the case $\varrho_{r h}=\varrho_{r h}^{\min }$ (long reheating). The dashed curves are obtained from the approximate relation (153). The red (upper) one for $N^{*}=\bar{N}_{\max }^{*}=59$ (instant reheating), and the blue (lower) one for $N^{*}=\bar{N}_{\text {min }}^{*}=57$ (long reheating). The dashed straight line corresponds to the relation (155), approximately valid in the region $\frac{2}{3 N^{*}}<1+w_{\mathrm{DE}}^{0} \ll 1$. Note that it does not exactly correspond to the straight line in Fig. 4, which would not correspond to a straight line in the present plot. The horizontal line and the shaded regions correspond to the observational mean value and the $1 \sigma$ and $3 \sigma$ confidence intervals, cf. (105).

$$
\begin{aligned}
\alpha_{\zeta} \simeq & \frac{48 w_{\mathrm{DE}}^{a}}{\left(-4+9 \delta_{\mathrm{DE}}^{0}\right)^{3}}\left(\left(-4+9 \delta_{\mathrm{DE}}^{0}\right) \operatorname{coth}\left(\frac{6 N^{*} \delta_{\mathrm{DE}}^{0}}{4-9 \delta_{\mathrm{DE}}^{0}}\right)\right. \\
& \left.+6 N^{*} \delta_{\mathrm{DE}}^{0} \sinh ^{-2}\left(\frac{6 N^{*} \delta_{\mathrm{DE}}^{0}}{4-9 \delta_{\mathrm{DE}}^{0}}\right)\right),
\end{aligned}
$$

connecting the running $\alpha_{\zeta}$ of the scalar spectral index to the equation of state parameter $w_{\mathrm{DE}}^{0}$ of dark energy and its rate of change $w_{\mathrm{DE}}^{a}$, defined through

$$
w_{\mathrm{DE}}(a)=w_{\mathrm{DE}}^{0}+w_{\mathrm{DE}}^{a} \ln \left(a / a_{0}\right) .
$$

While the first-order consistency condition (153) should become testable in the near future, a the test of the secondorder relation (154) will be more challenging.

We have mentioned previously that the parameter region where $n_{s}$ is well-approximated by the asymptotic linear in $\xi_{\chi}$ is not excluded by observations. In terms of $\delta_{\mathrm{DE}}$, this region is given by $\delta_{\mathrm{DE}} \ll 1$ and $1<\frac{6 N^{*} \delta_{\mathrm{DE}}^{0}}{4-9 \delta_{\mathrm{DE}}^{0}} \simeq \frac{3}{2} N^{*} \delta_{\mathrm{DE}}^{0}$, in which the relation (153) becomes approximately

$$
-3\left(1+w_{\mathrm{DE}}^{0}\right) \simeq\left(n_{s}-1\right), \text { for } \frac{2}{3 N^{*}}<\delta_{\mathrm{DE}} \ll 1,
$$

which can equivalently be written as a relation between "first orders" in the early and the late Universe:

$$
\frac{d \ln \varrho_{\mathrm{DE}}^{0}}{d \ln a} \simeq \frac{d \ln P_{\zeta}}{d \ln k} .
$$

Whether this is a fundamental consequence of SI or just a coincidence remains yet unclear. Note that if relation (156) should hold, it would not only imply that the deviation $\delta_{\mathrm{DE}}^{0}$ of dark energy from a cosmological constant is proportional to the deviation $n_{s}$ of the primordial spectrum from the scale-invariant one. In fact, if we could take it at face value, it would imply a concrete value for the present abundance of dark energy: $F\left(\Omega_{\mathrm{DE}}\right)=1 / 2 \Rightarrow \Omega_{\mathrm{DE}}=$ 0.739 , surprisingly close to the observed value. In the same region of parameter space, also the respective second-order quantities are proportional to each other, again for $\Omega_{\mathrm{DE}}=0.74$,

$$
3 w_{\mathrm{DE}}^{a} \simeq \alpha_{\zeta},
$$

or equivalently

$$
\frac{d^{2} \ln \varrho_{\mathrm{DE}}^{0}}{(d \ln a)^{2}} \simeq \frac{d^{2} \ln P_{\zeta}}{(d \ln k)^{2}} .
$$

Let us stress again that the links between the observables $n_{s}$ and $\alpha_{\zeta}$, related to inflation, and $w_{\mathrm{DE}}^{0}$ and $w_{\mathrm{DE}}^{a}$, related to dark energy, are nontrivial predictions of the present model. They relate two a priori totally independent periods and allow us to use the measurable observables from CMB anisotropies to make predictions for the widely unknown DE sector. On the other hand, one should bear in mind that these results rely on several important assumptions. In particular, the functional relations are based on the requirement that the Jordan frame potential has a flat direction $(\beta=0)$.

\section{B. Dark-energy constraints on initial conditions}

Let us now show how the obtained results justify the assumption we made about inflation taking place in the scale-invariant region. From Eq. (152), we infer that $\varrho_{\mathrm{QE}}$ is dominated by the potential energy contribution, and, hence, $\varrho_{\mathrm{QE}} \simeq V_{\mathrm{QE}}(\tilde{\rho})$. This allows us to deduce from the observational value $\Omega_{\mathrm{QE}}^{0}=\Omega_{\mathrm{DE}}^{0} \simeq 0.74$ today's value of $\tilde{\rho}$,

$$
\tilde{\rho}_{0} \simeq-\frac{1}{4 \gamma} M_{P} \ln \left(\gamma^{4} \frac{\Lambda_{\mathrm{eff}}}{\Lambda_{0}}\right),
$$

where we have defined an effective cosmological constant as

$$
\Lambda_{\text {eff }} \equiv 3 M_{P}^{2} H_{0}^{2} \Omega_{\mathrm{DE}}^{0} \simeq 10^{-120} M_{P}^{4} .
$$

Now, numerical simulations show that the field $\tilde{\rho}$ has been almost constant from the end of inflation till today. Therefore, the value of $\tilde{\rho}_{0}$ provides an order of magnitude estimate for the value of $\tilde{\rho}$ at the end of inflation. During the analysis of inflation, we have made the assumption that the whole period of observable inflation, i.e. the last $\sim 60$ e-folds, took place in the scale-invariant region, where $v_{1}$, 
$v_{2} \ll 1$, and, hence, $\rho \simeq$ cst. . We can now check this assumption by computing $v_{1}$ and $v_{2}$, cf. Eqs. (53) and (54) at $\rho \simeq \rho^{*} \simeq \rho_{\text {end }} \simeq \gamma \tilde{\rho}_{0}$. Using Eq. (159) and working in the usual approximation $\xi_{\chi} \ll 1$ and $\xi_{h} \gg 1$, we obtain

$$
\begin{aligned}
& v_{1} \simeq \frac{144 \xi_{\chi}^{2} \xi_{h}^{2}}{\lambda} \frac{\Lambda_{\text {eff }}}{M_{P}^{4}} \frac{1}{\sin ^{4} \theta}, \\
& v_{2} \simeq \frac{24 \xi_{\chi} \xi_{h}^{2}}{\lambda} \frac{\Lambda_{\text {eff }}}{M_{P}^{4}} \frac{1}{\sin ^{2} \theta \cos \theta} .
\end{aligned}
$$

From Eqs. (112) and (114), we have $\theta_{\text {end }} \simeq 2 * 3^{1 / 4} \sqrt{\xi_{\chi}}$ and $\theta^{*} \simeq \arccos \left(e^{-4 \xi_{\chi} N^{*}}\right)$. Evaluating $v_{1}$ and $v_{2}$ for values $\xi_{\chi}$, $\xi_{h}$, and $N^{*}$ of the orders of magnitude found in Sec. III D and $0.1<\lambda<1$, we find that for the whole interval $\theta_{\text {end }}<$ $\theta<\theta^{*}, v_{1}, v_{2} \lll 1$, and, hence, that the deviation from exact scale invariance is negligible. This justifies a posteriori the neglecting of $\Lambda_{0}$ during inflation. Let us note that this conclusion is not altered if one takes into account the slight change of the scalar fields between the end of inflation and today. The change of $\tilde{\rho}$ during the reheating oscillations and during the thawing quintessence stage are of the percent level.

In Sec. III D, we have seen that, for a successful description of inflation, the initial conditions for the scalar fields need to satisfy $\theta_{\text {in }}>\theta^{*}$, respectively, $\frac{h_{\text {in }}}{\chi_{\text {in }}} \geq \sqrt{\frac{1+6 \xi_{\chi}}{1+6 \xi_{h}}} \tan \theta^{*}$. We recall that, for typical values $\xi_{\chi}=0.005, \xi_{h}=65000$, and $N^{*}=58$, one obtains $\frac{h_{\text {in }}}{\chi_{\text {in }}} \gtrsim 0.005$. The observational value for $\Omega_{\mathrm{DE}}^{0} \simeq 0.74$ (respectively, Eq. (159)), together with the knowledge that the field $\rho$ remains almost constant from horizon crossing during inflation until today, allows us to further restrict the region of allowed initial conditions (cf. Fig. 7). Namely, if $\tilde{\rho}$ is alone responsible for dark energy, and as long as the initial conditions lie in the scale-invariant region $\left(v_{1}, v_{2} \ll 1\right)$, the relation (159) yields approximately the initial value for the field $\rho$, i.e.

$$
\rho_{\text {in }} \simeq \rho^{*} \simeq \rho_{\text {end }} \simeq \gamma \tilde{\rho}_{0} \simeq-\frac{M_{P}}{4 t i} \ln \left(\gamma^{4} \frac{\Lambda_{\text {eff }}}{\Lambda_{0}}\right) \text {. }
$$

In terms of the original variables, this corresponds to a relation between $\chi_{\text {in }}$ and $h_{\text {in }}$ given by $\left(\xi_{\chi} \ll 1\right.$ and $\left.\xi_{h} \gg 1\right)$ :

$$
\frac{\chi_{\text {in }}^{2}}{\Lambda_{0}^{1 / 2}}+6 \xi_{h} \frac{h_{\text {in }}^{2}}{\Lambda_{0}^{1 / 2}} \simeq \frac{1}{\xi_{\chi}} \frac{M_{P}^{2}}{\Lambda_{\text {eff }}^{1 / 2}} \sim 10^{60} .
$$

Together with the bound $h_{\text {in }} / \chi_{\text {in }} \gtrsim 10^{-3}$, this shows that initial conditions have to approximately satisfy $h_{\text {in }} / \Lambda_{0}^{1 / 4} \gtrsim 10^{30}$. Hence, the initial value of $h$ has to be much larger than the scale $\Lambda_{0}^{1 / 4}$. For $\tilde{\rho}$ to exactly produce the observed abundance of dark energy, the initial values have to be chosen precisely on a line in the $(\rho, \theta)$-, respectively, the $(\chi, h)$-plane. This tuning of initial conditions is commonly referred to as the Cosmic
Coincidence Problem (see e.g. [83]). ${ }^{24}$ Our model does not alleviate this problem with respect to other quintessence models. In fact, if one allows for an additional dark-energy component, present if $\beta>0$, the set of acceptable initial conditions extends to an infinite region. In that case, while the fine-tuning issue does not concern the initial conditions, the parameter $\beta$ has to be finely tuned. Hence, in either case some "fine-tuning" is needed. At this point, it should be recalled that, although the Cosmic Coincidence Problem is an undesirable feature, it is not a consistency problem and, therefore, does not invalidate this and other models of dynamical dark energy.

Finally, we can briefly comment on the case of initial conditions lying in the region where $\Lambda_{0}$ cannot be neglected. Initial conditions lie in this region $\left(v_{2}>1\right)$ whenever $\theta_{\text {in }}$ is sufficiently close to $\pi / 2$, respectively, when $h_{\text {in }} / \chi_{\text {in }}$ is sufficiently big. Note, however, that as a consequence of condition (162) and for typical parameter values, this only happens for extreme values $\pi / 2-\theta_{\text {in }} \lesssim 10^{-112}$, respectively, $h_{\text {in }} / \chi_{\text {in }} \gtrsim 10^{109}$. In that region, $\rho$ is no longer constant. The E-frame potential (29) becomes dominated by the term proportional to $\Lambda_{0}$, i.e. $\tilde{U}(h, \chi) \simeq \tilde{V}_{\Lambda_{0}}(h, \chi)=$ $\frac{M_{P}^{4} \Lambda_{0}}{\left(\xi_{\chi} \chi^{2}+\xi_{h} h^{2}\right)^{2}}$. The effect of this potential is to drive the scalar fields to larger values of $\chi$ and $h$, respectively, larger values of $\rho$, before they enter into the scale-invariant region. Qualitatively, this means that, in the non-scale-invariant region, the line of successful initial conditions is no longer given by Eq. (162) (respectively, Eq. (163)) but turns towards the origin. Still, the discussion related to the Cosmic Coincidence Problem equally applies to initial conditions in this region.

\section{CONCLUSIONS}

We have considered a minimal scale-invariant extension of the standard model, nonminimally coupled to gravity, including a scalar dilaton. All mass scales at the classical level, including the Planck scale and the Electroweak scale, are induced by the spontaneous breaking of the scale invariance. The physical dilaton is almost massless but hardly affects particle physics phenomenology. Our findings rely on SI, both at the classical and the quantum level $[2,3]$. The replacement of standard general relativity by unimodular gravity gives rise to an arbitrary constant in the equations of motion, which, in a minimally coupled theory, would play the role of a cosmological constant. However, due to the nonminimal couplings between the scalar and the gravitational sectors, this constant gives rise to a nontrivial run-away potential for the dilaton. As a consequence, the dilaton can play the role of a quintessence field, responsible for a late dark-energy dominated stage. For appropriate values of the free parameters and initial

\footnotetext{
${ }^{24}$ For a discussion of the fine-tuning issue in the particular case of a quintessence field with an exponential potential, see [79].
} 
conditions, the constructed model presents a rich cosmological phenomenology, providing mechanisms both for inflation and dark energy.

We find that the amplitude $P_{\zeta}$ of CMB anisotropies depends mainly on the ratio $\xi_{h} / \sqrt{\lambda}$, while the spectral tilt $n_{s}$, the associated running $\alpha_{\zeta}$, as well as the scalar-totensor ratio $r$ depend mainly on $\xi_{\chi}$. The observational limits on $P_{\zeta}$ and $n_{s}$ put bounds on $\xi_{h} / \sqrt{\lambda}$ and $\xi_{\chi}$, which in turn provide the bounds $\alpha_{\zeta} \lesssim-0.00015$ and $r \gtrsim$ 0.0009. In addition, the model predicts the bounds $n_{s}<$ 0.97, $\alpha_{\zeta}>-0.0006$, and $r<0.0033$, which are obtained in the limit $\xi_{\chi} \rightarrow 0$ and correspond to the predictions of the Higgs-Inflation model of [1]. The confrontation of these bounds with the results of the Plack satellite mission will constitute an important test of the Higgs-dilaton model.

Neither SI nor unimodular gravity forbid the existence of a quartic term $\beta \chi^{4}$ in the Jordan frame, which would correspond to a proper cosmological constant in the Einstein frame. However, the parameter choice forbidding such a term $(\beta=0)$ appears to be specially interesting, both from the cosmological and the quantum theory point of view. For this choice, the dilaton alone is responsible for dark energy. The associated equation of state parameter $w_{\mathrm{DE}}^{0}$ is found to practically depend on $\xi_{\chi}$ only. This has the interesting consequence that the spectral index $n_{s}$ can be expressed as $n_{s}=n_{s}\left(w_{\mathrm{DE}}^{0}\right)$, thus relating an observable from the very early Universe to an observable of the present Universe. For a particular parameter region, this relation takes the simple form $-3\left(w_{\mathrm{DE}}^{0}+1\right) \simeq\left(n_{s}-1\right)$. The observational bound on $n_{s}$ translates into a bound $0 \leq 1+w_{\mathrm{DE}}^{0} \lesssim 0.02$, which might be tested by future experiments. Further, we were able to derive a relation between the running of the spectral index $\alpha_{\zeta}$ and the rate of change $3 w_{\mathrm{DE}}^{a}$ of the equation of state parameter. Notice that for the dilaton to provide the measured abundance of dark energy, initial conditions have to be finely tuned. Hence, as is the case for all quintessence models, the Cosmological Coincidence Problem remains unsolved.

\section{ACKNOWLEDGMENTS}

We thank Julien Lesgourgues and Andrei Linde for helpful discussions and useful comments. J. G. B. thanks the Institute of Theoretical Physics in Geneva for its hospitality. J.R. thanks EPFL for its hospitality. We also acknowledge financial support from the Madrid Regional Government (CAM) under the Program No. HEPHACOS P-ESP-00346 and MICINN under Grant No. AYA200913936-C06-06. We also participate in the ConsoliderIngenio 2010 PAU (CSD2007-00060), as well as in the European Union Marie Curie Network UniverseNet under Contract No. MRTN-CT-2006-035863. J. R. would like to acknowledge financial support from UAM/CSIC. The work of M.S. and D.Z. was supported by the Swiss National Science Foundation and by the Tomalla Foundation.

\section{APPENDIX A: HIGGS-DILATON INFLATION IN THE JORDAN FRAME}

This appendix is devoted to the study of the inflationary trajectories in the Jordan frame. We perform an analytical study of the trajectories of the scalar fields during slow roll and compare it with the results of an exact numerical computation in Jordan and Einstein representations. The numerical computation in the Einstein frame is performed in the way described in [55] and takes into account the nonminimal kinetic terms in Eq. (25). At the classical level, different frames just correspond to different choices of variables, and, therefore, the final physical results should not differ. Notice, however, the different units used in every frame. For homogeneous fields, $h=h(t)$ and $\chi=\chi(t)$, the conformal transformation of the metric from the Jordan to the Einstein frame (22) depends only on time $\Omega=\Omega(t)$ and implies a redefinition of the cosmic time, $d \tilde{t}=\Omega(t) d t$, as well as the scale factor, $\tilde{a}(\tilde{t})=$ $\Omega(t) a(t)$, in the Einstein frame. The associated Hubble rate should be also redefined as

$$
\tilde{H} \equiv \frac{1}{\tilde{a}} \frac{d \tilde{a}}{d \tilde{t}}=\frac{H}{\Omega}\left(1+\frac{\Omega^{\prime}}{\Omega}\right),
$$

where the prime denotes derivatives with respect to the number of e-folds $N$ in the Jordan frame. Relation (A1) allows us to easily obtain a useful relation between the number of e-folds computed in both frames,

$$
\Delta \equiv \frac{d N}{d \tilde{N}}=1-\frac{d \ln \Omega}{d \tilde{N}} .
$$

Integrating this equation from the initial field configuration $\phi_{0}$ at the beginning of inflation, we get

$$
\tilde{N}-N=\ln \frac{\Omega(\phi)}{\Omega\left(\phi_{0}\right)} \leq 0 .
$$

As expected, the number of e-folds is not an invariant under conformal transformations. However, the difference between the two frames turns out to be practically irrelevant during the inflationary stage. To obtain an upper bound, we focus on the value at the end of inflation, $\ln \left(\Omega_{\text {end }} / \Omega_{0}\right)$, where the discrepancy between $\tilde{N}$ and $N$ is larger. As we saw in Sec. III A 2, the inflationary are well described by ellipses with constant radius, $r_{0}^{2} \equiv\left(1+6 \xi_{h}\right) h_{0}^{2}+\left(1+6 \xi_{\chi}\right) \chi_{0}^{2}$. Here, $h_{0}$ and $\chi_{0}$ are the initial values for the Higgs and dilaton fields, respectively. Let us assume that they are roughly equal. In this case, it is possible to relate the initial and final amplitude of the $h$ field to obtain

$$
\frac{h_{\mathrm{end}}}{h_{0}} \simeq \sqrt{\frac{6 \xi_{\chi}}{1+12 \xi_{\chi}}},
$$

where we have used $\xi_{h} \gg \xi_{\chi}$ as well as the approximate relation among the field amplitudes at the end of inflation $\chi \simeq \sqrt{\frac{\xi_{h}}{\xi_{\chi}}} h$. Taking into account Eq. (A4), we 
obtain $\Omega_{\text {end }} / \Omega_{0} \simeq \sqrt{\frac{12 \xi_{X}}{1+12 \xi_{\chi}}}$, which corresponds, for a typical value $\xi_{\chi}=0.005$, to

$$
\left|\frac{N-\tilde{N}}{N}\right| \leq 2 \% \text {. }
$$

It can be shown numerically that, during most of the inflationary stage, the difference among the number of e-folds defined in both frames (A2) is indeed quite smaller than the previous bound. Given the small difference between the number of e-folds defined in Jordan and Einstein frames, we will from now on identify $N=\tilde{N}$. Let us now consider the Higgs-dilaton Lagrangian density in the Jordan frame (19). We will assume that the initial values of the fields are such that they evolve within the scale-invariant region, in which the $\Lambda_{0}$ term in Eq. (21) can be neglected. Far away from the valleys of the potential, the contribution of terms proportional to $\alpha \sim \mathcal{O}\left(10^{-30}\right)$ can also be safely ignored. The Klein-Gordon equations of motion for homogeneous scalar fields are then given by

$$
\ddot{\phi}^{a}+3 H \dot{\phi}^{a}+V^{a}-\frac{1}{2} f^{, a} R=0,
$$

where $R$ is the Ricci scalar, given by $R=6\left(\dot{H}+2 H^{2}\right)$. In the Jordan frame, field-space indices are raised and lowered with the Euclidean metric $\delta_{i j}$. The two Friedmann equations can be written as

$$
\begin{gathered}
3 H^{2} f(\phi)=\frac{1}{2} \dot{\phi}^{a} \dot{\phi}_{a}+V(\phi)-3 H \partial_{0} f(\phi), \\
f(\phi) R=-3\left(\partial_{0}^{2}+3 H \partial_{0}\right) f(\phi)-\dot{\phi}^{a} \dot{\phi}_{a}+4 V(\phi) .
\end{gathered}
$$

If we assume the fields to be homogenous during inflation, together with the standard slow-roll approximation, $\dot{\phi}^{a} \dot{\phi}_{a} \ll V, \ddot{\phi}^{a} \ll V^{a}$, and $\ddot{\phi}^{a} \ll H \dot{\phi}^{a}$, the equations of motion for the scalar fields (A6) expressed in terms of the number of e-folds $N$, become

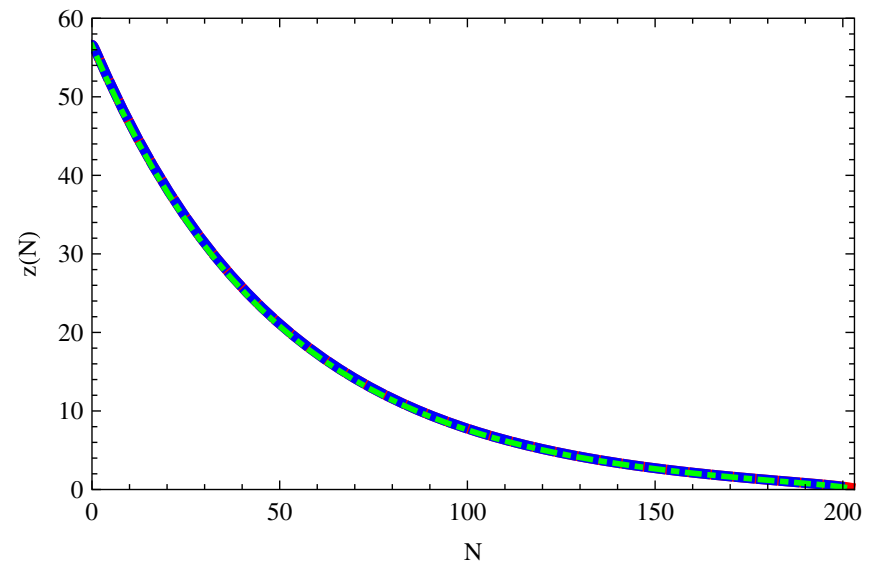

$$
3 H^{2} \phi^{a \prime} \simeq-V^{, a}+\frac{1}{2} f^{a} R, 《
$$

while the Friedmann equations (A7) and (A8) simplify, respectively, to (note that $\dot{f}=H f^{\prime}$ )

$$
\begin{gathered}
V \simeq 3 H^{2}\left(f+f^{\prime}\right), \\
f R \simeq 4 V-9 H^{2} f^{\prime} .
\end{gathered}
$$

In the last equation, we have assumed extended slow-roll conditions, namely $1+6 \xi_{a} \dot{\phi}^{a} \ll V(\phi)$ and $1+6 \xi_{a} \dot{\phi}^{a} \ll$ $H \dot{f}(\phi)$, which should be checked numerically a posteriori. Equations (A10) and (A11) imply that the Ricci scalar can be approximated as $R \simeq 12 H^{2}\left(1+f^{\prime} /(4 f)\right)$, which does not correspond to the usual approximation $\dot{H} \ll H^{2}$. Although it can be checked numerically that the contribution of the extra term $f^{\prime} /(4 f)$ is indeed very small, it must be explicitly maintained to preserve the conservation of the dilatational current in the slow-roll approximation. Indeed, combining Eqs. (A9)-(A11), we obtain the field-space constraint

$$
\left(1+6 \xi_{\chi}\right) \chi \chi^{\prime}+\left(1+6 \xi_{h}\right) h h^{\prime} \simeq 0,
$$

which, as shown in Sec. III A 2, gives rise (in those cases in which the $\Lambda_{0}$ term can be neglected) to inflationary trajectories that can be properly described as ellipses in field space. Therefore, it will be useful to apply the same strategy of Sec. III A 2 and rewrite the problem in terms of polar coordinates $(r, z)$, defined as

$$
\begin{aligned}
r^{2} & \equiv\left(1+6 \xi_{h}\right) h^{2}+\left(1+6 \xi_{\chi}\right) \chi^{2}, \\
z & \equiv \sqrt{\frac{\left(1+6 \xi_{h}\right)}{\left(1+6 \xi_{\chi}\right)}} \frac{h}{\chi},
\end{aligned}
$$

where $z=\tan \theta$, cf. Eq. (43). The evolution equation for the previous variables can be computed making use of Eqs. (A9)-(A11) to obtain

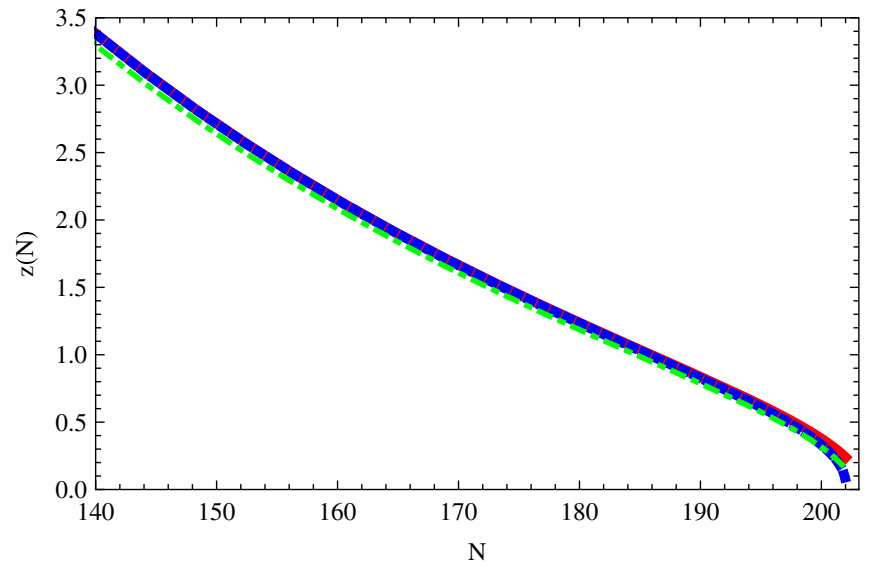

FIG. 10 (color online). Evolution of the angular variable $z$ as a function of the number of e-folds $N$ and detailed view of the last 60 e-folds. The green (dotted-dashed) lines represent the approximate slow-roll solutions given by Eq. (A15), while the red (solid) and blue (dashed) curves correspond to the result of an exact numerical computation performed in the Jordan and Einstein frames, respectively. 

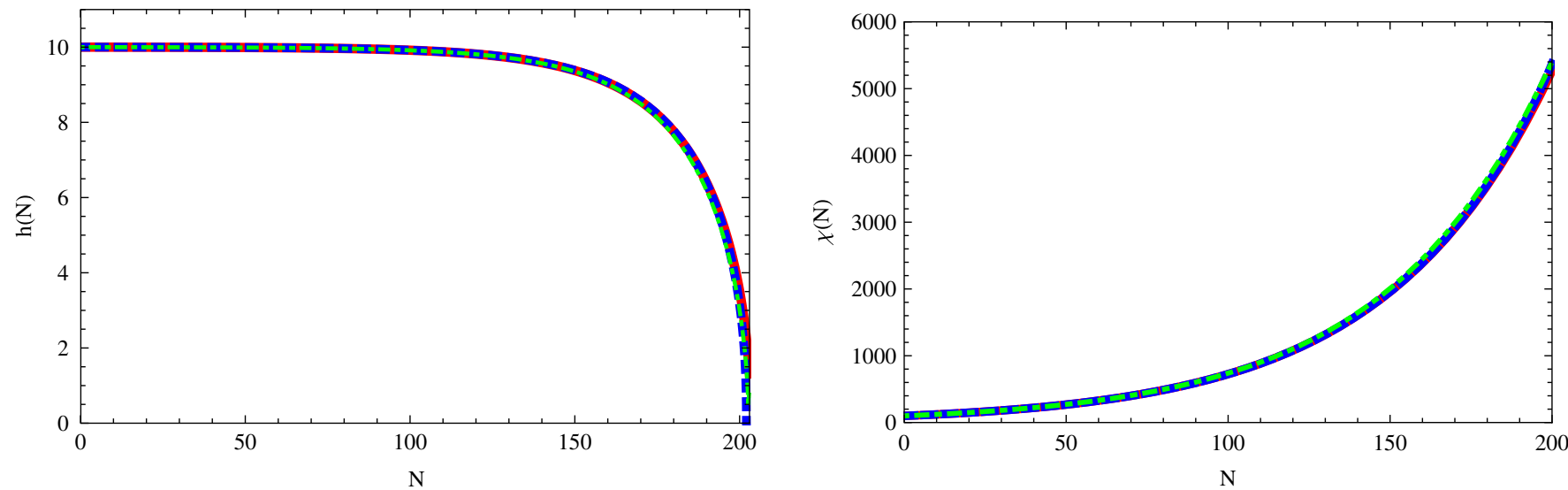

FIG. 11 (color online). Evolution of the Higgs $h$ and dilaton $\chi$ fields as a function of the number of e-folds $N$. The green (dotteddashed) lines represent the approximate slow-roll solutions given by Eq. (A16), while the red (solid) and blue (dashed) curves are exact numerical results in the Jordan and Einstein frames, respectively.

$$
r^{\prime} \simeq 0, \quad \frac{z^{\prime}}{z} \simeq-4 \xi_{\chi} \frac{z^{2}+\varsigma}{z^{2}+\varsigma+2 \xi_{\chi}}\left(1+\frac{1}{z^{2}}\right)
$$

where s depends on the couplings $\xi_{h}, \xi_{\chi}$ and is given by Eq. (47). The previous equations can be easily solved to obtain the evolution of the radial and angular coordinates with the number of e-folds:

$$
r=r_{0}, \frac{\left(1+z^{2}\right)^{1-2 \xi_{\chi}}\left(z^{2}+\varsigma\right)^{2 \xi_{\chi}}}{\left(1+z_{0}^{2}\right)^{1-2 \xi_{\chi}}\left(z_{0}^{2}+\varsigma\right)^{2 \xi_{\chi}}}=e^{-8 \xi_{\chi} N}
$$

where $r_{0}$ and $z_{0}$ stand for the initial values of the fields. The comparison between the slow-roll solution (A15) for the $z$ variable and the exact solutions obtained numerically in Jordan and Einstein frames is shown in Fig. 10. Notice that, as pointed out above, we have identified the number of e-folds computed in Jordan with that computed Einstein frame, $N \simeq \tilde{N}$, given the small difference between the two during the whole inflationary period. As expected, the evolution of the dimensionless quantity $z$ does not depend on the chosen frame. Making use of Eq. (A15), it is also possible to compute the corresponding values of the original Higgs and dilaton fields, which, in terms of the $z$ variable, can be written as

$$
\begin{aligned}
& h(N)=\frac{r(N)}{\sqrt{1+6 \xi_{h}}}\left(1+z^{-2}(N)\right)^{-1 / 2}, \\
& \chi(N)=\frac{r(N)}{\sqrt{1+6 \xi_{\chi}}}\left(1+z^{2}(N)\right)^{-1 / 2} .
\end{aligned}
$$

The comparison with the numerical solutions is shown in Fig. 11.
[1] M. Shaposhnikov and D. Zenhausern, Phys. Lett. B 671, 187 (2009).

[2] M. Shaposhnikov and D. Zenhausern, Phys. Lett. B 671, 162 (2009).

[3] F. Englert, C. Truffin, and R. Gastmans, Nucl. Phys. B117, 407 (1976).

[4] F. L. Bezrukov and M. Shaposhnikov, Phys. Lett. B 659, 703 (2008).

[5] P. Jain and S. Mitra, Mod. Phys. Lett. A 22, 1651 (2007).

[6] P. Jain and S. Mitra, Mod. Phys. Lett. A 25, 167 (2010).

[7] D. S. Salopek, J. R. Bond, and J. M. Bardeen, Phys. Rev. D 40, 1753 (1989).

[8] K. Nakamura et al. (Particle Data Group), J. Phys. G 37, 075021 (2010).

[9] T. Asaka, S. Blanchet, and M. Shaposhnikov, Phys. Lett. B 631, 151 (2005).
[10] T. Asaka and M. Shaposhnikov, Phys. Lett. B 620, 17 (2005).

[11] P. A. M. Dirac, Proc. R. Soc. A 165, 199 (1938).

[12] D. Blas, M. Shaposhnikov, and D. Zenhausern, Phys. Rev. D 84, 044001 (2011).

[13] M. E. Shaposhnikov and I. I. Tkachev, Phys. Lett. B 675, 403 (2009).

[14] M.E. Shaposhnikov and F. V. Tkachov, arXiv:0905.4857.

[15] M. Shaposhnikov, in Workshop on Astroparticle Physics: Current Issues (APCI07), Budapest, Hungary, 2007 (unpublished), arXiv:0708.3550.

[16] B. Allen and A. Folacci, Phys. Rev. D 35, 3771 (1987).

[17] P. Bizon and A. Rostworowski, Phys. Rev. Lett. 107, 031102 (2011).

[18] I. Antoniadis, J. Iliopoulos, and T. N. Tomaras, Phys. Rev. Lett. 56, 1319 (1986). 
[19] N. C. Tsamis and R.P. Woodard, Phys. Lett. B 301, 351 (1993).

[20] N. C. Tsamis and R. P. Woodard, Ann. Phys. 238, No. 1, 1 (1995).

[21] I. Antoniadis, P. O. Mazur, and E. Mottola, New J. Phys. 9, 11 (2007).

[22] A. M. Polyakov, Nucl. Phys. B834, 316 (2010).

[23] J.J. van der Bij, H. van Dam, and Y.J. Ng, Physica (Amsterdam) 116A, 307 (1982).

[24] F. Wilczek, Phys. Rep. 104, 143 (1984).

[25] A. Zee, Proceedings of 20th Annual Orbis Scientiae, Miami, Florida, 1983 (Plenum, NY, 1985), 211.

[26] W. Buchmuller and N. Dragon, Phys. Lett. B 207, 292 (1988).

[27] W. G. Unruh, Phys. Rev. D 40, 1048 (1989).

[28] S. Weinberg, Rev. Mod. Phys. 61, 1 (1989).

[29] M. Henneaux and C. Teitelboim, Phys. Lett. B 222, 195 (1989).

[30] W. Buchmuller and N. Dragon, Phys. Lett. B 223, 313 (1989).

[31] F. Bezrukov, D. Gorbunov, and M. Shaposhnikov, J. Cosmol. Astropart. Phys. 06 (2009) 029.

[32] J. Garcia-Bellido, D. G. Figueroa, and J. Rubio, Phys. Rev. D 79, 063531 (2009).

[33] F. Bezrukov, D. Gorbunov, M. Shaposhnikov, arXiv:1106.5019.

[34] C. Wetterich, Nucl. Phys. B302, 668 (1988).

[35] B. Ratra and P. J. E. Peebles, Phys. Rev. D 37, 3406 (1988).

[36] P. G. Ferreira and M. Joyce, Phys. Rev. D 58, 023503 (1998).

[37] R. R. Caldwell and E. V. Linder, Phys. Rev. Lett. 95, 141301 (2005).

[38] S. R. Coleman, J. Wess, and B. Zumino, Phys. Rev. 177, 2239 (1969).

[39] N. Makino and M. Sasaki, Prog. Theor. Phys. 86, 103 (1991).

[40] M.E. Peskin and D. V. Schroeder, An Introduction to Quantum Field Theory (Addison-Wesley, Reading, Massachussetts, 1995) p. 842.

[41] J. Garcia-Bellido and D. Wands, Phys. Rev. D 52, 6739 (1995).

[42] J. Garcia-Bellido and D. Wands, Phys. Rev. D 53, 5437 (1996).

[43] F. Di Marco, F. Finelli, and R. Brandenberger, Phys. Rev. D 67, 063512 (2003).

[44] C. M. Peterson and M. Tegmark, Phys. Rev. D 83, 023522 (2011).

[45] D. H. Lyth, in Summer School in High Energy Physics and Cosmology, ICTP, 1993 (unpublished), arXiv:astro-ph/ 9312022.

[46] D. J. Schwarz, C. A. Terrero-Escalante, and A. A. Garcia, Phys. Lett. B 517, 243 (2001).

[47] A. Sakharov, Zh. Eksp. Teor. Fiz. 49, 245 (1965).

[48] V. Lukash, Pis'ma Zh. Eksp. Teor. Fiz. 31, 631 (1980); [JETP Lett. 31, 596 (1980)].

[49] V.F. Mukhanov and G. V. Chibisov, Pis'ma Zh. Eksp. Teor. Fiz. 33, 549 (1981) [JETP Lett. 33, 532 (1981)].

[50] G. V. Chibisov and V. F. Mukhanov, Mon. Not. R. Astron. Soc. 200, 535 (1982).

[51] D. H. Lyth, Phys. Rev. D 31, 1792 (1985).
[52] V. F. Mukhanov, H. A. Feldman, and R. H. Brandenberger, Phys. Rep. 215, 203 (1992).

[53] J. M. Bardeen, Phys. Rev. D 22, 1882 (1980).

[54] H. Kodama and M. Sasaki, Prog. Theor. Phys. Suppl. 78, 1 (1984).

[55] C. Ringeval, Lect. Notes Phys. 738, 243 (2008).

[56] M. Sasaki, Prog. Theor. Phys. 76, 1036 (1986).

[57] V.F. Mukhanov, Zh. Eksp. Teor. Fiz. 94N7, 1 (1988) [Sov. Phys. JETP 67, 1297 (1988)].

[58] C. Gordon, D. Wands, B. A. Bassett, and R. Maartens, Phys. Rev. D 63, 023506 (2000).

[59] A. R. Liddle and D. H. Lyth, Phys. Rep. 231, 1 (1993).

[60] T. Chiba and M. Yamaguchi, J. Cosmol. Astropart. Phys. 01 (2009) 019.

[61] S. M. Leach, A. R. Liddle, J. Martin, and D. J. Schwarz, Phys. Rev. D 66, 023515 (2002).

[62] F. Finelli and R.H. Brandenberger, Phys. Rev. D 62, 083502 (2000).

[63] S. Tsujikawa and B.A. Bassett, Phys. Lett. B 536, 9 (2002).

[64] E. Komatsu et al. (WMAP Collaboration), Astrophys. J. Suppl. Ser. 192, 18 (2011).

[65] J.C. Mather, D. J. Fixsen, R. A. Shafer, C. Mosier, and D. T. Wilkinson, Astrophys. J. 512, 511 (1999).

[66] A. O. Barvinsky, A. Y. Kamenshchik, and A. A. Starobinsky, J. Cosmol. Astropart. Phys. 11 (2008) 021.

[67] A. De Simone, M. P. Hertzberg, and F. Wilczek, Phys. Lett. B 678, 1 (2009).

[68] F. L. Bezrukov, A. Magnin, and M. Shaposhnikov, Phys. Lett. B 675, 88 (2009).

[69] F. Bezrukov and M. Shaposhnikov, J. High Energy Phys. 07 (2009) 089.

[70] F. Bezrukov, A. Magnin, M. Shaposhnikov, and S. Sibiryakov, J. High Energy Phys. 01 (2011) 016.

[71] S. Ferrara, R. Kallosh, A. Linde, A. Marrani, and A. Van Proeyen, Phys. Rev. D 82, 045003 (2010).

[72] S. Ferrara, R. Kallosh, A. Linde, A. Marrani, and A. Van Proeyen, Phys. Rev. D 83, 025008 (2011).

[73] R. Kallosh, L. Kofman, A. D. Linde, and A. Van Proeyen, Classical Quantum Gravity 17, 4269 (2000); 21, 5017(E) (2004).

[74] Planck Collaboration, arXiv:astro-ph/0604069.

[75] C. Wetterich, Nucl. Phys. B302, 645 (1988).

[76] E. J. Copeland, M. Sami, and S. Tsujikawa, Int. J. Mod. Phys. D 15, 1753 (2006).

[77] R. J. Scherrer and A. A. Sen, Phys. Rev. D 77, 083515 (2008).

[78] E. J. Copeland, A. R. Liddle, and D. Wands, Phys. Rev. D 57, 4686 (1998).

[79] U. J. Lopes Franca and R. Rosenfeld, J. High Energy Phys. 10 (2002) 015.

[80] S. Sen, A. A. Sen, and M. Sami, Phys. Lett. B 686, 1 (2010).

[81] J. Annis et al. (DES Collaboration), arXiv:astro-ph/ 0510195; See also http://www.darkenergysurvey.org.

[82] A. Refregier, A. Amara, T.D. Kitching, A. Rassat, R. Scaramella, J. Weller, arXiv:1001.0061; See also http:// sci.esa.int/euclid.

[83] S. Weinberg, in Dark Matter 2000, Marina del Rey, California, 2000 (unpublished), arXiv:astro-ph/0005265. 